\title{
Carving out the space of open-string S-matrix
}

\author{
Yu-tin Huang, ${ }^{a, b}$ Jin-Yu Liu, ${ }^{a}$ Laurentiu Rodina ${ }^{c}$ and Yihong Wang ${ }^{a, d}$ \\ ${ }^{a}$ Department of Physics, National Taiwan University, \\ No. 1, Sec. 4, Roosevelt Road, Taipei 10617, Taiwan \\ ${ }^{b}$ Physics Division, National Center for Theoretical Sciences, National Tsing-Hua University, \\ No. 101, Section 2, Kuang-Fu Road, Hsinchu, Taiwan \\ ${ }^{c}$ Institut de Physique Theorique, Universite Paris Saclay, CEA, CNRS, \\ F-91191 Gif-sur-Yvette, France \\ ${ }^{d}$ Hangzhou Institute for Advanced Study, University of Chinese Academy of Sciences, \\ Hangzhou, Zhejiang, 310024, China \\ E-mail: yutinyt@gmail.com, k5438777@gmail.com, \\ laurentiu.rodina@gmail.com, yihong.w2@gmail.com
}

ABSTRACT: In this paper, we explore the open string amplitude's dual role as a space-time S-matrix and a 2D holomorphic CFT correlation function. We pursue this correspondence in two directions. First, beginning with a general disk integrand dressed with a KobaNielsen factor, we demonstrate that exchange symmetry for the factorization residue of the amplitude forces the integrand to be expandable on $\mathrm{SL}(2, \mathrm{R})$ conformal blocks. Furthermore, positivity constraints associated with unitarity imply the $\operatorname{SL}(2, \mathrm{R})$ blocks must come in linear combinations for which the Virasoro block emerges at the "kink" in the space of solutions. In other words, Virasoro symmetry arises at the boundary of consistent factorization. Next, we consider the low energy EFT description, where unitarity manifests as the EFThedron in which the couplings must live. The existence of a worldsheet description implies, through the Koba-Nielsen factor, monodromy relations which impose algebraic identities amongst the EFT couplings. We demonstrate at finite derivative order that the intersection of the "monodromy plane" and the four-dimensional EFThedron carves out a tiny island for the couplings, which continues to shrink as the derivative order is increased. At the eighth derivative order, on a three-dimensional monodromy plane, the intersection fixes the width of this island to around 1.5\% (of $\zeta(3))$ and $0.2 \%$ (of $\zeta(5)$ ) with respect to the toroidally compactified Type-I super string answer. This leads us to conjecture that the four-point open superstring amplitude can be completely determined by the geometry of the intersection of the monodromy plane and the EFThedron.

KEYwords: Conformal Field Models in String Theory, Scattering Amplitudes

ArXiv EPrint: 2008.02293 


\section{Contents}

1 Introduction 1

2 Consistent factorization and the emergence of Virasoro symmetry 7

$\begin{array}{ll}2.1 & \text { Global blocks from exchange symmetry } \\ & 10\end{array}$

2.2 Non-negativity and the Virasoro block 13

3 Review of monodromy relations and the EFThedron $\quad 17$

$\begin{array}{ll}3.1 & \text { The monodromy plane } \\ \end{array}$

$\begin{array}{ll}3.2 & \text { The EFThedron } \\ & 20\end{array}$

4 Intersection of monodromy plane and the EFThedron 22

4.1 The absence of isolated massless poles 23

$\begin{array}{ll}4.2 \text { Combined constraints } & 23\end{array}$

4.2.1 The $k=3$ geometry 24

$\begin{array}{lll}4.2 .2 & \text { The } k=4 \text { geometry } & 26\end{array}$

$\begin{array}{ll}4.2 .3 & \text { Peeking from beyond } k=4 \\ 28\end{array}$

4.3 Closed string EFT from KLT 31

5 Conclusions and outlook $\quad 32$

$\begin{array}{ll}\text { A Expanding coefficient } & 34\end{array}$

B Virasoro block $\quad 34$

$\begin{array}{ll}\text { C Bicolor ordered amplitudes } & \mathbf{3 5}\end{array}$

$\begin{array}{lll}\text { C.1 BCJ and monodromy plane } & 35\end{array}$

$\begin{array}{lll}\text { C.2 Combined constraints for bicolor ordered amplitude } & 37\end{array}$

$\begin{array}{ll}\text { D Checking a corollary of the main conjecture } & 38\end{array}$

\section{Introduction}

String theory amplitudes can be viewed as living in the intersection of two sets of consistency conditions: on the one hand they are subject to the usual analyticity constraints of the space-time S-matrix, on the other, as two-dimensional CFT correlators they must have a consistent operator product expansion (OPE). At four-points both sets of consistency systems are amenable to a bootstrap analysis, where a spectacular modern revival has been seen for the CFT bootstrap [1] (for a review see [2-4]), and more recently for scattering amplitudes [5-10]. 
The interplay between space-time factorization and the structure of the worldsheet integrand has been one of the major undertones in new formulations of field theory and string theory scattering amplitudes. Indeed, the Cachazo-He-Yuan (CHY) [11-13] formulation of massless scattering amplitudes are given as an integral over the moduli space of a Riemann sphere, where the "scattering equation" ensures that space-time factorization is projected into worldsheet factorization. In the modern representation of open string amplitudes, where it is written as a product of a field-theory amplitude with its $\alpha^{\prime}$ corrections, as demonstrated for the superstring [14-16] as well as bosonic and heteorotic strings [17, 18], it can be viewed as a UV completion of solutions to consistent massless factorization. Even more recently, consistent massless factorization that extrapolates between the $\alpha^{\prime} \rightarrow 0$ limit and finite $\alpha^{\prime}$ naturally led to the realization of stringy canonical forms whose generalization are not necessarily string theory amplitudes [19]. This leads us to ask: must a worldsheet representation that enjoys unitary massless and massive factorization be necessarily given by a CFT four-point function that respects the full Virasoro symmetry?

On the other hand, UV unitarity leads to non-trivial bounds on the low energy effective field theory (EFT) couplings. This was famously explored for the positivity of the leading four-derivative coupling stemming from the optical theorem in [20]. Extensions to higher order derivatives, and away from the forward limit, were explored in subsequent works [21-24], with a complete geometric definition identified as the EFThedron [25]. These are general unitarity constraints that do not require the presence of a worldsheet. ${ }^{1}$ Thus it is then natural to seek out features of the worldsheet that has a distinctive projection in the EFThedron.

In this paper, we wish to study the projection in both directions. The arena is the following ansatz for an open string amplitude

$$
A(s, t) \sim \int_{0}^{1} d z z^{\alpha^{\prime} 2 k_{3} \cdot k_{4}}(1-z)^{\alpha^{\prime} 2 k_{2} \cdot k_{3}} f(z),
$$

where $f(z)$ is some function such that $z^{\Delta} f(z)$ is analytic near $z=0$ for some finite $\Delta$, and $k_{i}$ are $d$-dimensional momenta. We use $s=-\left(k_{1}+k_{2}\right)^{2}, t=-\left(k_{1}+k_{4}\right)^{2}$, and $u=-\left(k_{1}+k_{3}\right)^{2}$, so that $A(s, t) \equiv A(1234)$ is an ordered amplitude with only $s$ and $t$ channel poles. Importantly, we will proceed by being completely agnostic about the world sheet theory, and any specific form of $f(z)$. Instead, in the first part of the paper we will derive the global conformal invariance as well as Virasoro symmetry of the worldsheet theory as contained by $f(z)$, by imposing exchange symmetry and unitarity of the space time S-matrix.

In the other direction, we will again start from a general $f(z)$, and study the low energy expansion of eq. (1.1). By imposing unitarity constraints and the monodromy relations implied by the Koba-Nielsen factor $z^{\alpha^{\prime} 2 k_{3} \cdot k_{4}}(1-z)^{\alpha^{\prime} 2 k_{2} \cdot k_{3}}$, we will find that the low energy expansion must match the open string amplitude. Demanding eq. (1.1) satisfies monodromy relations amounts to generalizing $f(z)$ to gain non-trivial $s, t$ dependence, while not sourcing any non-trivial monodromy. This can be viewed as the amplitude for the vacuum state of the compactified string on a product geometry $R^{1, d-1} \otimes M$, where $M$ is compact. A canonical example will be toroidal compactification of flat space string amplitude.

\footnotetext{
${ }^{1}$ They were recently studied in the context of string amplitudes in [26].
} 
Note that even though eq. (1.1) might seem to severely limit the space of EFT's we can consider, in fact any general EFT can be embedded in that form with a suitable choice for $f(z)$. For example, choosing an $s-t$ symmetric polynomial:

$$
f(z)=\sum_{i=0} c_{a} z^{a}(1-z)^{a}
$$

We can perform the integral to obtain

$$
A(s, t)=\sum c_{k} \frac{\Gamma\left[\alpha^{\prime} s+a\right] \Gamma\left[\alpha^{\prime} t+a\right]}{\Gamma\left[\alpha^{\prime}(s+t)+2 a\right]}
$$

Expanding this at low energy in the $\alpha^{\prime} \rightarrow 0$ limit we obtain

$$
A(s, t)=c_{0}\left(\frac{1}{s}+\frac{1}{t}\right) \alpha^{\prime-1}+\left(c_{1}+\frac{c_{2}}{6}+\frac{c_{3}}{30}+\ldots\right)+\mathcal{O}\left(\alpha^{\prime}\right)
$$

and so we can express any general EFT of the type

$$
A(s, t)=\sum g_{k, q} s^{k-q} t^{q}
$$

simply by solving for the coefficients $c_{a}$. Practically, the Koba-Nielsen factors act as a UV dressing that can be applied to any EFT in order to control its high energy behavior. However, as is well known that the "intercept" $a$ in eq. (1.2) is severely constrained by unitarity. Thus we expect that by combining eq. (1.1) with the constraints from the EFThedron, the space for allowed $f(z)$ and hence EFT coefficients, are severely constrained.

Space-time constraints on the worldsheet. We first consider constraints on the function $f(z)$ imposed by the fact that $A(s, t)$, as a space-time S-matrix, must factorize in a way that is consistent with unitarity and Lorentz symmetry. The latter implies that the residue must be expandable on the Gegenbauer polynomials ${ }^{2}$

$$
\left.\operatorname{Res}[A(s, t)]\right|_{s \rightarrow m^{2}}=\sum_{\ell} \mathcal{C}_{\ell} \frac{G_{\ell}^{d}(\cos \theta)}{s-m^{2}}
$$

while the former implies linear and quadratic bounds on $\mathcal{C}_{\ell}$. In particular, labeling the mass of the external legs as $i_{1}, i_{2}, i_{3}$ and $i_{4}$, unitarity implies

- (i) $\mathcal{C}_{\ell}$ is symmetric under $i_{1} \leftrightarrow i_{2}, i_{3} \leftrightarrow i_{4}$ exchange.

- (ii) $\mathcal{C}_{\ell}$ must be positive when $i_{1}=i_{4}$ and $i_{2}=i_{3}$.

- (iii) For distinct masses, the $\mathcal{C}_{\ell}$ 's satisfy the Schwarz inequality:

$$
\mathcal{C}_{\ell}\left(i_{1}, i_{2}, i_{2}, i_{1}\right) \mathcal{C}_{\ell}\left(i_{3}, i_{4}, i_{4}, i_{3}\right)-\left(\mathcal{C}_{\ell}\left(i_{1}, i_{2}, i_{3}, i_{4}\right)\right)^{2} \geq 0 .
$$

\footnotetext{
${ }^{2}$ Recall that the Gegenbauer polynomials are orthogonal polynomials that form irreducible representations of $\mathrm{SO}(d-1)$. They are given by the following generating function

$$
\frac{1}{\left(1-2 r \cos \theta+r^{2}\right)^{\frac{d-3}{2}}}=\sum_{\ell} r^{\ell} G_{\ell}^{d}(\cos \theta) .
$$
}




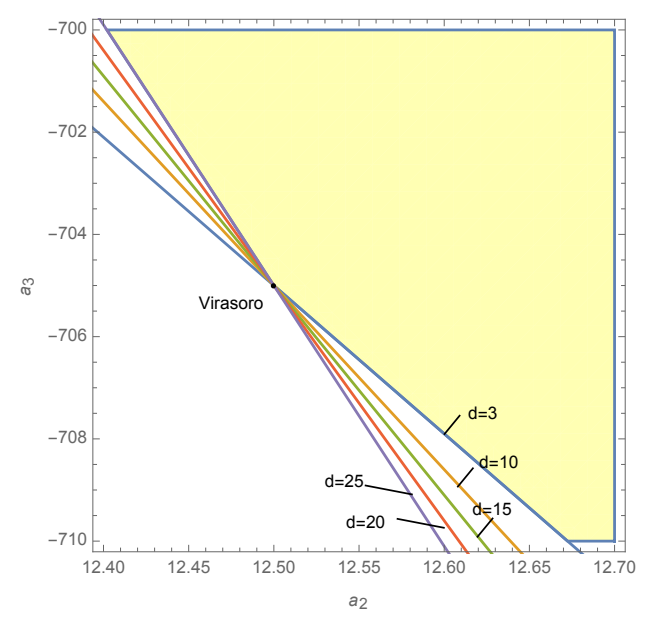

Figure 1. The plot for the allowed solution for an ansatz for $\chi_{4}(2.41)$ under (ii) and (iii). For illustrative purposes we have set $\left(a_{1}, a_{4}\right)$ to the Virasoro block value $\left(a_{1}=9880, a_{4}=5\right)$, and plotted $a_{2}, a_{3}$. The lines denote the boundaries carved out by (iii) as a function of the space-time dimension. The point in the figure is the Virasoro value.

Since $z^{\Delta} f(z)$ is analytic near the origin, the singularity of the amplitude arises from when the exponents of the Koba-Nielson factor become negative integers. This allows us to easily extract the $s$-channel factorization residues and consider the consequences of the above constraints on the power series coefficients of $f(z)$. We find that (i) alone is sufficient to show that $f(z)$ must be given by linear combinations of $\mathrm{SL}(2, \mathrm{R})$ global conformal blocks, expressed as hypergeometric functions of the type ${ }_{2} F_{1}(\Delta, \Delta, 2 \Delta, z)$. Conditions (ii) and (iii) impose further constraints. In particular, we will show that for these constraints to hold for arbitrary positive external masses, the global blocks whose conformal dimensions differ by integers must be grouped into a new function of the form:

$$
z^{\Delta}{ }_{2} F_{1}(\Delta, \Delta, 2 \Delta, z)+\sum_{q=1}^{\infty} \chi_{q} z^{\Delta+q}{ }_{2} F_{1}(\Delta+q, \Delta+q, 2 \Delta+2 q, z),
$$

with their relative coefficients $\chi_{q}$ bounded by (ii) and (iii). Using this criteria we find that $\chi_{i}$ with $i=1,2,3$ can be completely determined as the solution that saturates the bounds in (ii) and (iii). For $\chi_{4}$ where things become more subtle, we can plot the allowed region and demonstrate that the Virasoro block lives at the kink of the region, as shown in figure 1. Thus we see that worldsheet Virasoro symmetry emerges at the boundary of consistent space-time factorization. It remains an interesting open question whether this allowed region can be further reduced by extra constraints. Assuming further properties such as unitarity of the CFT, it is known that scale invariance implies conformal invariance. However, it is not clear for example that the spacetime unitarity we are imposing on the Smatrix image of this CFT requires the CFT itself to be unitary, and so conformal invariance is not guaranteed.

Worldsheet image on space-time S-matrix. We then consider the projection of constraints in the opposite direction. We ask what is the image of the string worldsheet on the 
S-Matrix? Looking back at eq. (1.1), one consequence is that the Koba-Nielsen factor implies the following monodromy relation amongst amplitudes of different orderings [27-29]:

$$
A(s, u)+e^{i \pi s} A(s, t)+e^{-i \pi u} A(t, u)=0 .
$$

Note that it is known that monodromy relations, when combined with the asymptotic Regge behavior of string amplitudes, are sufficient to completely determine the four-particle amplitude [30]. In particular, the residue of the four-point amplitude is a single variable function, whose zeros on the real line can be completely determined by the monodromy relations. Using the precise form of the asymptotic Regge limit along with the precise intercept, one can conclude that no other zeros are present and the residue is fixed up to an overall constant. Note that it is important to impose the linear Regge trajectory. In fact it is has been shown long ago, that general principles of analyticity, crossing and linear Regge trajectory for the S-matrix can fix the four-point function to a sum of Veneziano amplitudes with kinematic dependent coefficients [31-35]. It is important to note that we will not assume the linear Regge trajectory, in particular its intercept, but merely the Froissart bound, which is a more general condition.

Here we would like to consider open strings in a more general context, for example on non-trivial compactified backgrounds. The ground-state scattering amplitude takes the form of eq. (1.1), with $f(z) \rightarrow f(z, s, t)$ being the four point function of some compact CFTs. Note that due to the presence of $f(z, s, t)$, the asymptotic Regge behavior will deviate from the usual flat space form, in particular in its intercept. Instead we consider the following assumptions

- (i) The UV completion respects Lorentz invariance and unitarity.

- (ii) There are no tachyonic states.

- (iii) The subtracted amplitude respects the Froissart bound [36].

- (iv) The amplitude satisfies the monodromy relation in eq. (1.9).

Here, (ii, iii) are necessary for the employment of the EFThedron constraints, which we will discuss shortly. The last assumption corresponds to $f(z, s, t)$ sourcing no extra monodromy. These conditions will be implemented from the EFT point of view, manifested as constraints for the EFT couplings. Taking the low energy limit and expanding the amplitude in Mandelstam invariants

$$
\left.A(s, t)\right|_{s, t \ll 1}=\text { (massless poles) }+\sum_{k, q \geq 0} g_{k, q} s^{k-q} t^{q},
$$

the monodromy relation imposes algebraic identities between the EFT couplings $g_{k, q}$, which importantly can also be amongst couplings of different mass dimensions. For instance, it fixes $g_{0,0}=\frac{\pi^{2}}{6}$, or $g_{3,1}=2 g_{3,0}-\frac{\pi^{2}}{6} g_{1,0}$. The remaining free parameters, like $g_{1,0}$ or $g_{3,0}$, define the "monodromy plane" in the space of EFT couplings. 
On the other hand, UV unitarity, Lorentz invariance and locality also constrain the space of allowed couplings to be inside the EFThedron [25]. Thus the image of the worldsheet inside the EFThedron is given by its intersection with the monodromy plane. Remarkably, we find that the intersection gives just a tiny allowed region for the independent EFT couplings. We will consider the EFThedron for four-dimensional space-time. For example, up to $k=4$ (eight derivative order), the monodromy plane is three-dimensional and parameterized by $g_{1,0}, g_{3,0}$ and $g_{4,1}$. Applying EFThedron constraints on this space, we obtain a finite intersection region displayed in gray in figure 2. The region is drastically reduced by requiring it to be uplifted into the $k=6$ geometry, for the cases when the four-dimensional monodromy plane intersects with the EFThedron. It is instructive to compare the width of the reduced red region to toroidally compactified type-I superstring amplitude. We find the coefficients must lie within a narrow region of the string result:

$$
\begin{aligned}
& \frac{g_{1,0}^{\max }-g_{1,0}^{\min }}{g_{1,0}^{\text {string }}}=\frac{1.20667-1.18890}{\zeta(3)} \approx 1.5 \%, \\
& \frac{g_{3,0}^{\max }-g_{3,0}^{\min }}{g_{3,0}^{\text {string }}}=\frac{1.03808-1.03594}{\zeta(5)} \approx 0.2 \%, \\
& \frac{g_{4,1}^{\max }-g_{4,1}^{\min }}{g_{4,1}^{\text {string }}}=\frac{0.05699-0.03560}{\left.\left(\pi^{6}-630 \zeta(3)^{2}\right) / 1260\right)} \approx 52.8 \% .
\end{aligned}
$$

Applying all $k=7$ and one $k=8$ constraints, using the FindInstance function in Mathematica we were able to further shrink to the region in figure $12 \mathrm{~b}$. In fact, by setting $\left(g_{1,0}, g_{3,0}, g_{4,1}\right)$ to string values $\left(\zeta(3), \zeta(5),\left(\pi^{6}-630 \zeta(3)^{2}\right) / 1260\right)$, we can search for solutions to the $k=7$ EFThedron constraints using FindInstance, obtaining the following values:

$$
\left(g_{5,0}, g_{6,1}, g_{7,0}, g_{7,2}\right)=(1.00834,0.00862,1.00202,0.00035),
$$

which match to the known string values up to four digits:

$$
\begin{aligned}
& \left(\zeta(7), \frac{\pi^{8}}{7560}-\zeta(3) \zeta(5), \zeta(9), \frac{180 \zeta(3)^{3}-2 \pi^{6} \zeta(3)-27 \pi^{4} \zeta(5)-540 \pi^{2} \zeta(7)+10080 \zeta(9)}{1080}\right) \\
& \quad=(1.00835,0.00865,1.00201,0.00032) .
\end{aligned}
$$

This analysis leads us to the conjecture that:

The geometry of intersection between the monodromy plane and the EFThedron yields the four-point massless amplitude of toroidally compactified Type-I superstring.

Note that this also implies that deviation from the superstring result must imply non-trivial monodromy factor arising from the four-point function of the compact CFT. We repeat the monodromy and unitarity study on one of such variation: the bicolour monodromy relation $\frac{u}{s} A(s, u)+e^{i \pi s} A(s, t)+e^{-i \pi u} \frac{t}{s} A(u, t)=0$, and achieve results similar to the single color amplitude. 


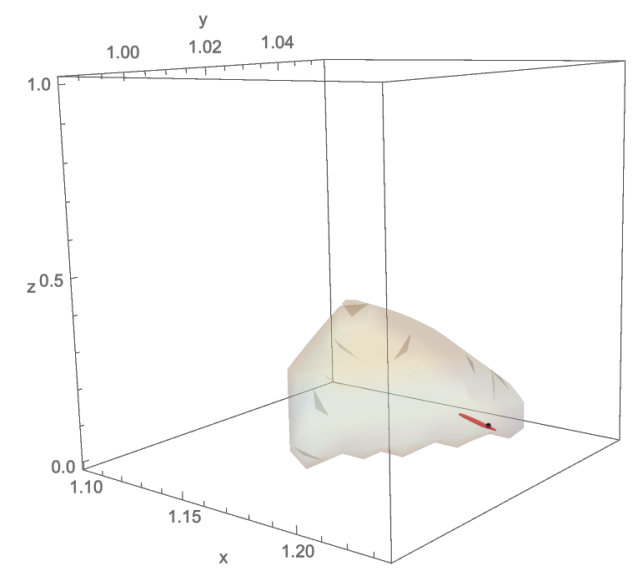

Figure 2. The gray region represents the three-dimensional intersection of the monodromy plane and EFThedron at eighth derivative order. The red region represents the region that can be projected from the four-dimensional geometry that would appear at ninth derivative order.

This paper is organized as follows. In section 2 we discuss the emergence of global conformal and Virasoro blocks from unitarity. Extra details are presented in appendix A and B. In section 3 we move on to review the monodromy relations and the EFThedron. In section 3.1 we first solve the monodromy condition perturbatively, and extract the relations imposed between different physical parameters, the monodromy plane. In section 3.2 we then review the EFThedron, the positive geometry in which EFT parameters must exist in order to satisfy unitarity and locality. In section 4 we apply EFThedron considerations on the remaining free parameters of the monodromy plane, and demonstrate how considering higher and higher order cyclic polytopes and Hankel matrices drastically reduces the allowed physical space, apparently converging to the open string amplitude. Appendix C contains the equivalent discussions for bi-color theory, while appendix D contains extra numerical checks of the conjecture. We end with conclusions and future directions in section 5 .

\section{Consistent factorization and the emergence of Virasoro symmetry}

In this section we ask the following question: suppose we have a four-point scalar amplitude that takes the form

$$
A(s, t)=\int_{0}^{1} d z z^{\alpha^{\prime} 2 k_{3} \cdot k_{4}}(1-z)^{\alpha^{\prime} 2 k_{2} \cdot k_{3}} f(z)
$$

where $\alpha^{\prime}$ is a normalization scale and we assume $f(z)$ is such that $z^{\Delta} f(z)$ is analytic near $z=0$ for some finite $\Delta$. We will not assume that $f(z)$ arises from the four-point function of a CFT, but aim to derive it. The form of (2.1) can be motivated from several fronts. Firstly, the kinematic dependence is completely contained in the Koba-Nielsen factor, which leads to exponential softness at $s,-t \gg 1$ à la Gross and Mende [37] (see [38] for open strings), as well as a linear trajectory for $s, t \gg 1$, which was shown to be universal in [39]. Secondly, one can consider this as an ansatz for the scattering of the vacuum state in $d$-dimensions, for string theory compactified on $R^{1, d-1} \otimes M_{d_{c}-d}$, where $d_{c}$ is the critical dimension. 
Since the kinematic dependence is all in the Koba-Nielsen factor, the amplitude can only develop singularities when this factor diverges, i.e. at the boundary of the integration region $z=0,1$. In other words, we will only have $s$ and $t$-channel singularities, implying that the amplitude has a prescribed ordering. This motivates us to re-express (2.1) as

$$
A(s, t)=\int_{0}^{1} d z z^{-s+i_{3}+i_{4}-2}(1-z)^{-t+i_{2}+i_{3}-2} f(z),
$$

where we denote the mass of each leg as $m_{a}^{2}=i_{a}-1$, where the -1 at this point is just convention. Note that we have set $\alpha^{\prime}=1$. We can extract the residue of the $s$-channel singularity by writing $f(z)$ as a series with generic real exponents in the neighborhood of $z=0$, which without loss of generality we write as

$$
f(z)=\sum_{p} c_{p} z^{p-i_{3}-i_{4}}
$$

This can be viewed as a sum of "dilatation blocks" weighed by coefficients $c_{p}$. For each individual block, the integral

$$
\int_{0}^{1} d z z^{-s-2}(1-z)^{-t+i_{2}+i_{3}-2} z^{p}
$$

will have $s$-channel poles at $p-1, p, p+1, \cdots$. This suggests that we collect the dilatation blocks that differ by integer exponents into a subset. To this end we consider

$$
f_{p}(z)=z^{p-i_{3}-i_{4}} D_{\left\{i_{a}\right\}}, \quad D_{\left\{i_{a}\right\}}=1+v_{1} z+v_{2} z^{2}+\cdots=1+\sum_{i=1}^{\infty} v_{i} z^{i} .
$$

Note that the relative coefficients $v_{i}$ are understood to be functions of $\left\{i_{a}, p\right\}$.

The $s$-channel residue is then given by evaluating the integral in (2.2) as a contour integral around $z=0$ for fixed $s$. We see that the $s$-channel singularity appears at $s=$ $n+p-1$, where $n$ is a non-negative integer, and the residue is given by

$$
\left.\frac{d^{n}}{d z^{n}}(1-z)^{t+i_{2}+i_{3}-2} D_{\left\{i_{a}\right\}}\right|_{z=0} \equiv \operatorname{Res}_{n}(t)
$$

Since $A(s, t)$ is a $d$-dimensional scalar scattering amplitude, we expand the residue function $\operatorname{Res}_{n}(t)$ in the Gegenbauer polynomial basis. This can be done by first converting $t$ to the center of mass scattering angle via:

$$
\cos \theta=\frac{\left(s+m_{1}^{2}-m_{2}^{2}\right)\left(s+m_{4}^{2}-m_{3}^{2}\right)-2 s\left(m_{1}^{2}+m_{4}^{2}-t\right)}{\sqrt{\left(s-m_{1}^{2}-m_{2}^{2}\right)^{2}-4 m_{1}^{2} m_{2}^{2}} \sqrt{\left(s-m_{3}^{2}-m_{4}^{2}\right)^{2}-4 m_{3}^{2} m_{4}^{2}}} .
$$

On the residue $s$ is set to $s=n+p-1$. Using this we can expand the residue on the $d$-dimensional Gegenbauer polynomials $G_{\ell}^{d}(\cos \theta)$ :

$$
\operatorname{Res}_{n}(\cos \theta)=\sum_{\ell} \mathcal{C}_{\ell}^{(n)} K^{\ell} G_{\ell}^{d}(\cos \theta)
$$




\begin{tabular}{|c|c|c|c|c|}
\hline $\mathcal{C}_{\ell}^{(n)}$ & $\ell=0$ & $\ell=1$ & $\ell=2$ & $\ell=3$ \\
\hline$n=0$ & 1 & 0 & 0 & 0 \\
\hline$n=1$ & $-\frac{\left(-i_{1}+i_{2}+p\right)\left(i_{3}-i_{4}+p\right)}{2 p}+v_{1}$ & $\frac{1}{2(-3+d)}$ & 0 & 0 \\
\hline$n=2$ & Appendix A & $-\frac{\left(i_{2}-i_{1}\right)\left(i_{3}-i_{4}+p+1\right)+(p+1)\left(i_{3}-i_{4}+p\right)}{4(d-3)(p+1)}+\frac{1}{2(d-3)} v_{1}$ & $\frac{1}{4(d-3)(d-1)}$ & 0 \\
\hline
\end{tabular}

Table 1. The explicit coefficient $\mathcal{C}_{\ell}^{(n)}$ of $\operatorname{Res}_{n}(t)$ in eq. (2.6) for $n=0,1,2$.

where $K$ is a kinematic factor given as:

$$
K=\frac{\sqrt{\left(n+p-m_{1}^{2}-m_{2}^{2}-1\right)^{2}-4 m_{1}^{2} m_{2}^{2}} \sqrt{\left(n+p-m_{3}^{2}-m_{4}^{2}-1\right)^{2}-4 m_{3}^{2} m_{4}^{2}}}{n+p-1} .
$$

In general the coefficients $\mathcal{C}_{\ell}^{(n)}$ will be given in terms of $\left\{i_{a}, d, p\right\}$, and $v_{i}$. For fixed $n$, $\mathcal{C}_{\ell}^{(n)}$ are non-vanishing for $\ell \leq n$, and they depend on $v_{i}$ with $i \leq n-\ell$. Take $\operatorname{Res}_{n}(t)$ in eq. (2.6) up to $n=2$ for example, expanding onto the Gegenbauer basis in eq. (2.8) leads to coefficients $\mathcal{C}_{\ell}^{(n)}$ listed in table $1 .^{3}$

Now since the residue function $\operatorname{Res}_{n}(t)$ must have an interpretation as the factorization of the four-point amplitude into the product of three-point amplitudes, it should have an equivalent representation as

$$
\operatorname{Res}_{n}(t)=\sum_{\ell} g_{\ell}^{i_{1} i_{2}} k_{12}^{\mu_{1}} k_{12}^{\mu_{2}} \cdots k_{12}^{\mu_{\ell}} \mathbf{P}_{\mu_{1} \mu_{2} \cdots \mu_{\ell} ; \nu_{1} \nu_{2} \cdots \nu_{\ell}} k_{34}^{\nu_{1}} k_{34}^{\nu_{2}} \cdots k_{34}^{\nu_{\ell}} g_{\ell}^{i_{3} i_{4}}
$$

where $k_{12}^{\mu}=k_{1}^{\mu}-k_{2}^{\mu}$ and $\mathbf{P}_{\mu_{1} \mu_{2} \cdots \mu_{\ell} ; \nu_{1} \nu_{2} \cdots \nu_{\ell}}$ is a degree $\ell$ polynomial in $\eta^{\mu \nu}$, that is symmetric and traceless in $\{\mu\}$ and $\{\nu\}$ separately. This implies the following constraints on the coefficients $\mathcal{C}_{\ell}^{(n)}$,

- (i) Under $i_{1} \leftrightarrow i_{2}, i_{3} \leftrightarrow i_{4}$ exchange, $\mathcal{C}_{\ell}^{(n)}$ is symmetric.

- (ii) For $i_{1}=i_{4}$ and $i_{2}=i_{3}, \mathcal{C}_{\ell}^{(n)}$ must be positive.

- (iii) For $i_{1} \neq i_{2} \neq i_{3} \neq i_{4}$, the $\mathcal{C}_{\ell}^{(n)}$ s satisfy the following quadratic Schwarz inequality:

$$
\mathcal{C}_{\ell}^{(n)}\left(i_{1}, i_{2}, i_{2}, i_{1}\right) \mathcal{C}_{\ell}^{(n)}\left(i_{3}, i_{4}, i_{4}, i_{3}\right)-\left(\mathcal{C}_{\ell}^{(n)}\left(i_{1}, i_{2}, i_{3}, i_{4}\right)\right)^{2} \geq 0
$$

The inequality (iii) arises from the fact that $\mathcal{C}_{\ell}^{(n)}$ should admit a representation as

$$
\begin{aligned}
& \mathcal{C}_{\ell}^{(n)}\left(i_{1}, i_{2}, i_{2}, i_{1}\right)=\sum_{a} g_{a i_{1} i_{2}} g_{a i_{1} i_{2}}=\left|\vec{g}_{i_{1} i_{2}}\right|^{2} \\
& \mathcal{C}_{\ell}^{(n)}\left(i_{1}, i_{2}, i_{3}, i_{4}\right)=\sum_{a} g_{a i_{1} i_{2}} g_{a i_{3} i_{4}}=\vec{g}_{i_{1} i_{2}} \cdot \vec{g}_{i_{3} i_{4}} \\
& \mathcal{C}_{\ell}^{(n)}\left(i_{3}, i_{4}, i_{4}, i_{3}\right)=\sum_{a} g_{a i_{3} i_{4}} g_{a i_{3} i_{4}}=\left|\vec{g}_{i_{3} i_{4}}\right|^{2},
\end{aligned}
$$

\footnotetext{
${ }^{3}$ At $n=0$ we only have $\mathcal{C}_{0}^{(0)}$, which is simply a constant proportional to $f(0)$.
} 
where $g_{a i j}$ is the coupling constant of some spin- $\ell$ state $a$ coupled to states $i$ and $j$. Note that the equality is satisfied if the $a=1$, i.e. the intermediate state is unique, or if there is a degeneracy and all the states couple with the same coupling.

Since $\mathcal{C}_{\ell}^{(n)}$ is defined with respect to the Gegenbauer polynomial, constraints (ii) and (iii) are dimension dependent. A Gegenbauer polynomial $G_{\ell}^{d_{1}}(\cos \theta)$ can be positively expanded on $G_{\ell}^{d_{2}}(\cos \theta)$ when $d_{1}>d_{2}$, therefore the space carved out by (ii) with $d_{1}$ must be inside that of $d_{2}$. For (iii), note that while eq. (2.12) implies eq. (2.11), the inverse is not true. Thus for an S-matrix to be consistent in $d_{1}$ dimensions, constraint (iii) must hold for all $d_{2}<d_{1}$. Thus it is necessary to compare and combine constraints of distinct dimensions. Because $\mathcal{C}_{\ell}^{(n)}$ is a function of the $v_{i}$, the above conditions are now translated into constraints on the function $f_{p}(z)$. In other words, if the integral formula in (2.1) is to yield a consistent space-time S-matrix, the power series of $f_{p}(z)$ must satisfy an infinite series of constraints!

In the following, we will demonstrate that condition (i) implies each $f_{p}(z)$ must be the global conformal block ${ }^{4}$

$$
f_{p}(z)=z^{p-i_{3}-i_{4}} F_{1}\left(p+\left(i_{2}-i_{1}\right), p+\left(i_{3}-i_{4}\right), 2 p, z\right),
$$

while (ii) and (iii) further require that the global blocks that differ by integer dimensions must be combined into further subsets:

$$
f_{p}(z)+\sum_{q=1} \chi_{q} f_{p+q}(z)
$$

with the Virasoro blocks living at the "boundary" of this subset. This is summarized in figure 3 .

\subsection{Global blocks from exchange symmetry}

The exchanging symmetry of $\mathcal{C}_{\ell}^{(n)}$ under $i_{1} \leftrightarrow i_{2}, i_{3} \leftrightarrow i_{4}$ imposes constraints on the form of $v_{i}$, as seen in table 1 . We will begin by assuming that $v_{n}$ takes the following factorized form:

$$
v_{n}=\frac{\mathcal{F}\left(i_{1}, i_{2}, p\right) \mathcal{F}\left(i_{3}, i_{4}, p\right)}{\mathcal{G}(p)} .
$$

\footnotetext{
${ }^{4}$ Note that there is an extra factor of $z^{-i_{3}-i_{4}}$, compared to the usual definition of global blocks, associated with the prefactors of the four-function which are partially canceled by the Koba-Nielsen factor. This can be traced back to the original form prior to $\mathrm{SL}(2, \mathrm{R})$ gauge fixing:

$$
A(s, t)=\int_{0}^{1} d z_{3} \prod_{i<j} z_{i j}^{2 k_{i} \cdot k_{j}} \frac{\left(\frac{z_{14}}{z_{24}}\right)^{i_{2}-i_{1}}\left(\frac{z_{14}}{z_{13}}\right)^{i_{3}-i_{4}}}{z_{12}^{i_{1}+i_{2}} z_{34}^{i_{3}+i_{4}}} \sum_{p} C_{p} z^{p}{ }_{2} F_{1}\left(p+\left(i_{2}-i_{1}\right), p+\left(i_{3}-i_{4}\right), 2 p, z\right) .
$$

Taking $\left(z_{1}, z_{2}, z_{3}, z_{4}\right)=(\infty, 1, z, 0)$ and including the gauge fixing factor we find

$$
(\infty)^{2\left(m_{1}^{2}-i_{1}+1\right)} \int_{0}^{1} d z_{3} z^{\alpha^{\prime} 2 k_{3} \cdot k_{4}}(1-z)^{\alpha^{\prime} 2 k_{2} \cdot k_{3}} \sum_{p} C_{p} z^{p-i_{3}-i_{4}}{ }_{2} F_{1}\left(p+\left(i_{2}-i_{1}\right), p+\left(i_{3}-i_{4}\right), 2 p, z\right) .
$$

The factor $\infty$ drops out if we have $m_{1}^{2}-i_{1}+1=0$, which is the expected relation between the mass and the $\mathrm{SL}(2, \mathrm{R})$ conformal dimension $m^{2}=h-1$.
} 


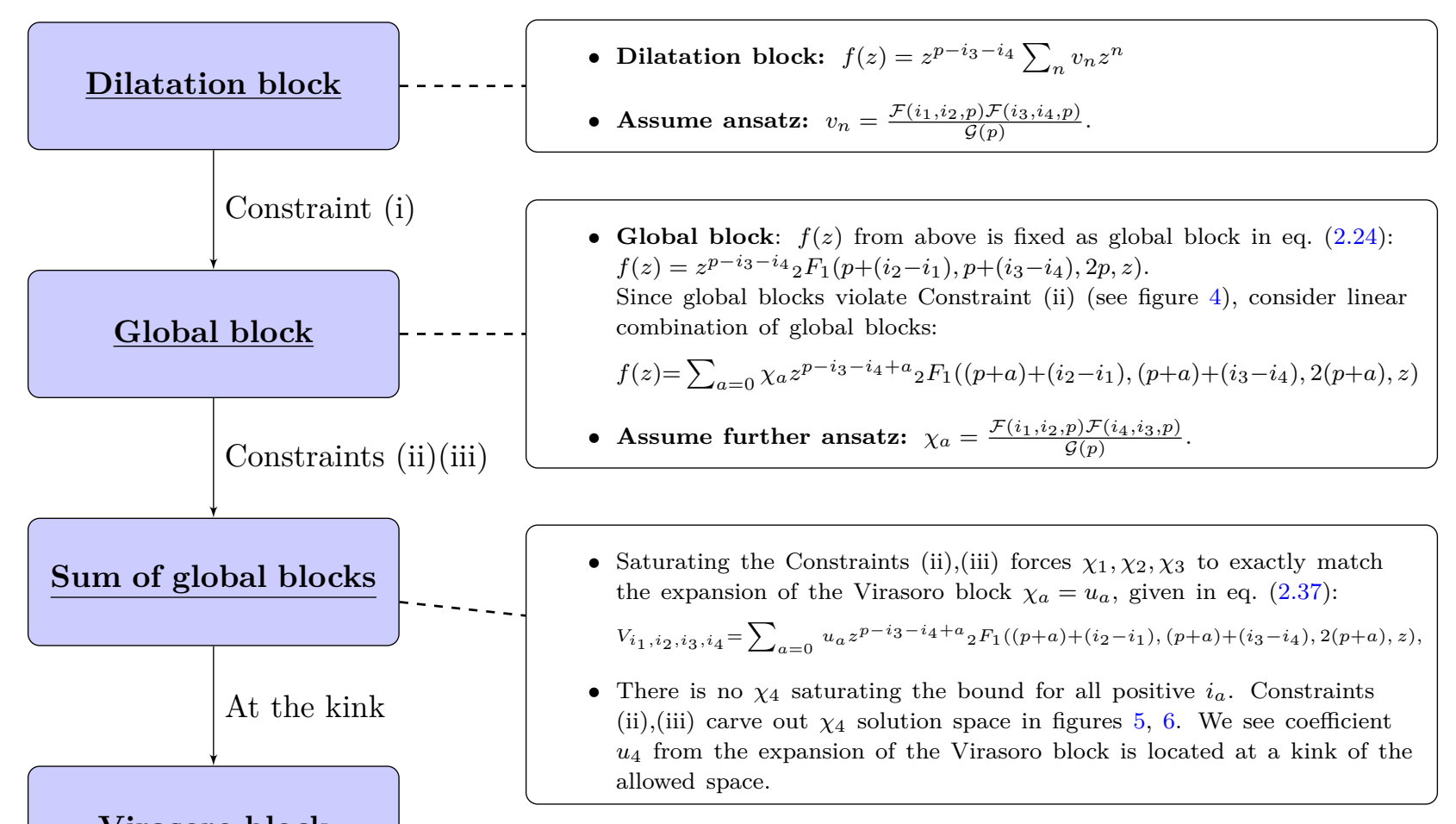

\section{Virasoro block}

Figure 3. Starting with the expression in eq. (2.2) and (2.5), constraint (i) forces the dilatation block to combine into global blocks. Imposing (ii) and (iii) requires the global blocks to be further combined, with the Virasoro blocks appearing at special "kinks" of the allowed space.

This form is motivated by the fact that it is associated with the product of two three-point functions. Note that the kinematic part of (2.10) suggests that for spin- $\ell$ exchange the residue is further symmetric under sole $i_{1} \leftrightarrow i_{2}$ exchange for $\ell \in$ even and anti-symmetric for $\ell \in$ odd (and similarly for $i_{3} \leftrightarrow i_{4}$ ). However, this conclusion is too hasty, as the coupling constants can also introduce compensating transformation properties, as in the case of structure constants of non-abelian algebra $f^{a b c}$. In light of this, we will only require that the residue has definite parity under the combined exchange $i_{1} \leftrightarrow i_{2}$ and $i_{3} \leftrightarrow i_{4}$.

Let us begin with $v_{1}$, which appears by itself in the $n=1$ scalar coefficient $\mathcal{C}_{0}^{(1)}$ and $n=2$ spin- 1 coefficient $\mathcal{C}_{1}^{(2)}$ in table 1 . Beginning with the ansatz

$$
v_{1}=\frac{\left(a_{1} i+a_{2} i_{2}+a_{3} p+a_{4} i_{1}^{2}+a_{5} i_{2}^{2}+a_{6} i_{1} i_{2}\right)\left(a_{1} i_{4}+a_{2} i_{3}+a_{3} p+a_{4} i_{4}^{2}+a_{5} i_{3}^{2}+a_{6} i_{3} i_{4}\right)}{a_{7}+a_{8} p+a_{9} p^{2}},
$$

by simply solving

$$
\mathcal{C}_{0}^{(1)}\left(i_{1}, i_{2}, i_{3}, i_{4}\right)-\mathcal{C}_{0}^{(1)}\left(i_{2}, i_{1}, i_{4}, i_{3}\right)=\mathcal{C}_{1}^{(2)}\left(i_{1}, i_{2}, i_{3}, i_{4}\right)-\mathcal{C}_{1}^{(2)}\left(i_{2}, i_{1}, i_{4}, i_{3}\right)=0,
$$

and requiring definite parity, we find a unique solution:

$$
v_{1}=\frac{\left(-i_{1}+i_{2}+p\right)\left(i_{3}-i_{4}+p\right)}{2 p} .
$$


Note that this leads to $\mathcal{C}_{0}^{(1)}=0$ and

$$
\mathcal{C}_{1}^{(2)}=-\frac{\left(i_{1}-i_{2}\right)\left(i_{3}-i_{4}\right)}{4(d-3) p(1+p)}
$$

i.e. $\mathcal{C}_{1}^{(2)}$ is antisymmetric under $i_{1} \leftrightarrow i_{2}, i_{3} \leftrightarrow i_{4}$ exchange respectively. We will come back to this property shortly.

Moving on to $v_{2}$, we begin with the ansatz

$$
\begin{aligned}
v_{2} & =\frac{\mathcal{F}\left(i_{1}, i_{2}, p\right) \mathcal{F}\left(i_{3}, i_{4}, p\right)}{\left(b_{1}+b_{2} p+b_{3} p^{2}\right)} \\
\mathcal{F}\left(i_{1}, i_{2}, p\right) & =a_{1} i_{1}^{2}+a_{2} i_{2}^{2}+a_{3} p^{2}+a_{4} i_{1} i_{2}+a_{5} i_{1} p+a_{6} i_{2} p+a_{7} i_{1}+a_{8} i_{2}+a_{9} p+a_{10} .
\end{aligned}
$$

Using $v_{1}$ in eq. (2.18), once again solving

$$
\mathcal{C}_{0}^{(2)}\left(i_{1}, i_{2}, i_{3}, i_{4}\right)-\mathcal{C}_{0}^{(2)}\left(i_{2}, i_{1}, i_{4}, i_{3}\right)=0
$$

while requiring that $\mathcal{C}_{0}^{(2)}$ has definite parity under individual exchange leads to

$$
v_{2}=\frac{\left(-i_{1}+i_{2}+p\right)\left(-i_{1}+i_{2}+p+1\right)\left(i_{3}-i_{4}+p\right)\left(i_{3}-i_{4}+p+1\right)}{4 p(2 p+1)} .
$$

This pattern continues, at each $n$, the symmetric property of the scalar coefficient $\mathcal{C}_{0}^{(n)}$ algebraically leads to a unique solution for $v_{n}$.

It is straightforward to see that the expressions (2.18) and (2.23) precisely match the $z$ expansion of the global conformal block, given for example in [40]:

$$
\begin{aligned}
& z^{p-i_{3}-i_{4}}{ }_{2} F_{1}\left(p+\left(i_{2}-i_{1}\right), p+\left(i_{3}-i_{4}\right), 2 p, z\right)= \\
& =z^{p-i_{3}-i_{4}}\left(1+\frac{\left(-i_{1}+i_{2}+p\right)\left(i_{3}-i_{4}+p\right)}{2 p} z+\frac{\left(-1+i_{2}+p\right)\left(1-i_{1}+i_{2}+p\right)\left(i_{3}-i_{4}+p\right)\left(1+i_{3}-i_{4}+p\right)}{4 p(1+2 p)} z^{2}\right)
\end{aligned}
$$

Thus we see that when interpreted as a space-time scattering amplitude, exchange symmetry of the residue directly leads to $f(z)$ in $(2.2)$ to be given by a sum over $\mathrm{SL}(2, \mathrm{R})$ global conformal blocks!

We have concluded that if the residue is to respect exchange symmetry, the "dilatation blocks" must be linearly combined into an $\mathrm{SL}(2, \mathrm{R})$ global conformal block. We now move on to the factorization constraints (ii) and (iii) in (2.11). Let us consider the simplest case where $i_{1}=i_{2}=i_{3}=i_{4}=h$. We display the four dimensional coefficient $\mathcal{C}_{0}^{(2)}$ for the global block $z^{p-2 h}{ }_{2} F_{1}(p, p, 2 p, z)$ as a function of $(p, h)$ in figure 4 . We can see that there are regions of conformal dimension both for the external $(h)$ and internal $(p)$ that violate positivity bounds. In general, at order $n$, the spin $n$ coefficient derived from the global block will automatically satisfy the factorization constraints (ii) and (iii) in (2.11), but cease to do so when $\ell<n$. This suggests that one must further consider linear combinations of global blocks, which we now turn to. 


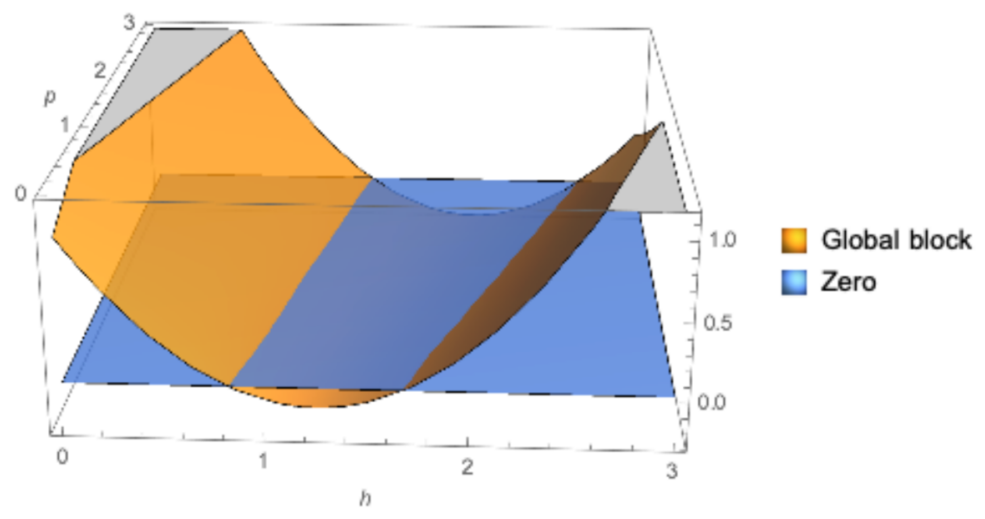

Figure 4. The scalar coefficient $\mathcal{C}_{0}^{(2)}$ associated with the global conformal block $(p, h)$. We see that there are regions of $(p, h)$ where the coefficient becomes negative, thus violating unitarity.

\subsection{Non-negativity and the Virasoro block}

We have seen that given a global block, for identical external states the residue coefficients can easily violate positivity. To remedy the situation, we consider linear combinations of global blocks. Note that since for any global block of conformal dimension $p$, the $s$-channel singularity occurs at $s=n+p-1$ with $n \in$ integer, we must consider linear combinations of blocks whose dimensions differ by integers. Thus we will be considering the following linear combination:

$$
f_{p}(z)=\sum_{a=0} \chi_{a} z^{p-i_{3}-i_{4}+a}{ }_{2} F_{1}\left((p+a)+\left(i_{2}-i_{1}\right),(p+a)+\left(i_{3}-i_{4}\right), 2(p+a), z\right),
$$

where $\chi_{0}=1$ and in general $\chi_{a}$ can be a function of $\left\{i_{1}, i_{2}, i_{3}, i_{4}, p, d\right\}$. Note that as the coefficients $\mathcal{C}_{\ell}^{(n)}$ are $d$ dependent, their positivity will translate to the $d$ dependence for $\chi_{a}$. Taking linear combinations of global blocks has the interpretation of taking linear combinations of $\mathrm{SL}(2, \mathrm{R})$ primaries, and thus the coefficients $\chi_{a}$ should once again take on factorized form reflecting their OPE nature. We therefore introduce an ansatz for $\chi_{a}$ of the form:

$$
\chi_{a}=\frac{\mathcal{F}\left(i_{1}, i_{2}, p\right) \mathcal{F}\left(i_{4}, i_{3}, p\right)}{\mathcal{G}(p)}
$$

We will be looking for polynomial solutions to $\mathcal{F}(i, j, p)$ that are of lowest degree in $i, j$. Next, we impose the factorization constraints in (2.11) (ii), (iii)

$$
\begin{array}{ll}
\text { (ii) } & \mathcal{C}_{\ell}^{(n)}\left(i_{1}, i_{2}, i_{2}, i_{1}\right)>0 \\
\text { (iii) } & \mathcal{C}_{\ell}^{(n)}\left(i_{1}, i_{2}, i_{2}, i_{1}\right) \mathcal{C}_{\ell}^{(n)}\left(i_{3}, i_{4}, i_{4}, i_{3}\right)-\left(\mathcal{C}_{\ell}^{(n)}\left(i_{1}, i_{2}, i_{3}, i_{4}\right)\right)^{2} \geq 0,
\end{array}
$$

which will carve out the solution space for $\chi_{a}$. Remarkably, we will find that the Virasoro block lives on the boundary of the solution space!

Let us analyze the constraints one order $n$ at a time. Recall that for fixed $n$ we have $\mathcal{C}_{\ell}^{(n)}$ with $\ell \leq n$. 
Order $\boldsymbol{n}=1$. Up to $n=1$, we have spin-0 and spin- 1 coefficients $\left(\mathcal{C}_{0}^{(1)}, \mathcal{C}_{1}^{(1)}\right)$, and only the scalar coefficient depends on $\chi_{1}$, given as:

$$
\mathcal{C}_{0}^{(1)}=\chi_{1}\left(i_{1}, i_{2}, i_{3}, i_{4}, p\right) .
$$

Now we ask if the scalar coefficient can saturate the bound in (2.28), for all positive external dimensions $\left(i_{1}, i_{2}, i_{3}, i_{4}\right)$. We find that a large family of solutions exists, of the form:

$$
\chi_{1}=\left(i_{1}-i_{2}\right)\left(i_{4}-i_{3}\right) \mathcal{F}(p) .
$$

The solution is minimal in the sense that it is lowest degree in external dimensions. With the above, (2.27) becomes:

$$
\mathcal{C}_{0}^{(1)}\left(i_{1}, i_{2}, i_{2}, i_{1}\right)=\left(i_{1}-i_{2}\right)^{2} \mathcal{F}(p) \geq 0 .
$$

Thus the "boundary" of (2.27) and (2.28) corresponds to $\mathcal{F}(p)=0=\chi_{1}$.

Order $\boldsymbol{n}=2$. At $n=2$, while we have spins up to $2, \chi_{2}$ only appears in the scalar coefficient $\mathcal{C}_{0}^{(2)}$. We will set $\chi_{1}=0$ and write down a minimal ansatz for $\chi_{2}$ in appendix B. We find that simply requiring the scalar coefficient to satisfy the equality in (2.28) for all external dimensions,

$$
\mathcal{C}_{0}^{(2)}\left(i_{1}, i_{2}, i_{2}, i_{1}\right) \mathcal{C}_{0}^{(2)}\left(i_{3}, i_{4}, i_{4}, i_{3}\right)-\left(\mathcal{C}_{0}^{(2)}\left(i_{1}, i_{2}, i_{3}, i_{4}\right)\right)^{2}=0, \quad \forall i_{1}, i_{2}, i_{3}, i_{4} \geq 0
$$

while respecting (2.27), the $\chi_{2}$ ansatz in (B.1) is reduced to one parameter function in ansatz space. The parameter will further restrict when we considering the positivity constraint at higher order $n$. It will be uniquely fixed and give us:

$\chi_{2}=\frac{\left(-3\left(i_{1}-i_{2}\right)^{2}+\left(i_{1}+i_{2}\right)+2 p\left(i_{1}+i_{2}\right)+(p-1) p\right)\left(-3\left(i_{4}-i_{3}\right)^{2}+\left(i_{4}+i_{3}\right)+2 p\left(i_{4}+i_{3}\right)+(p-1) p\right)}{2(1+2 p)\left(26-d+42 p-2 d p+16 p^{2}\right)}$.

All the detail can be found in appendix B.

Order $\boldsymbol{n}=3$. Let us now move to $n=3$, where we again begin with the scalar coefficient $\mathcal{C}_{0}^{(3)}$. The minimal ansatz for $\chi_{3}$ is given in appendix B. Setting $\chi_{1}=0$ and $\chi_{2}$ to (2.33), once again by imposing (2.27) and equality in (2.28) for $\mathcal{C}_{0}^{(3)}$,

$$
\mathcal{C}_{0}^{(3)}\left(i_{1}, i_{2}, i_{2}, i_{1}\right) \mathcal{C}_{0}^{(3)}\left(i_{3}, i_{4}, i_{4}, i_{3}\right)-\left(\mathcal{C}_{0}^{(3)}\left(i_{1}, i_{2}, i_{3}, i_{4}\right)\right)^{2}=0, \quad \forall i_{1}, i_{2}, i_{3}, i_{4} \geq 0
$$

the ansatz for $\chi_{3}$ can be completely fixed up to the ratio of parameters. The positivity in (2.27) for higher order coefficient will further restrict the ratio into fixed value and we have:

$$
\chi_{3}=-\frac{\left(i_{1}-i_{2}\right)\left(i_{3}-i_{4}\right)\left(-i_{1}+i_{1}^{2}-i_{2}+i_{2}^{2}-2 i_{1} i_{2}+p-i_{1} p-i_{2} p\right)\left(-i_{3}+i_{3}^{2}-i_{4}+i_{4}^{2}-2 i_{3} i_{4}+p-i_{3} p-i_{4} p\right)}{2 p(p+1)(p+2)\left(28-d+19 p-d p+3 p^{2}\right)} .
$$

Before moving on to $n=4$, let us compare $\left(\chi_{1}, \chi_{2}, \chi_{3}\right)$ in $(2.33)$ and $(2.35)$ to the Virasoro block expansion on the global blocks [40]:

$$
V_{i_{1}, i_{2}, i_{3}, i_{4}}=\sum_{a=0}^{\infty} u_{a} z^{p-i_{3}-i_{4}+a}{ }_{2} F_{1}\left((p+a)+\left(i_{2}-i_{1}\right),(p+a)+\left(i_{3}-i_{4}\right), 2(p+a), z\right),
$$


where

$$
\begin{aligned}
& u_{0}=1 \\
& u_{1}=0 \\
& u_{2}=\frac{\left(i_{1}-3 i_{1}^{2}+i_{2}-3 i_{2}^{2}+6 i_{1} i_{2}-p+2 i_{1} p+2 i_{2} p+p^{2}\right)\left(i_{3}-3 i_{3}^{2}+i_{4}-3 i_{4}^{2}+6 i_{3} i_{4}-p+2 i_{3} p+2 i_{4} p+p^{2}\right)}{2(1+2 p)(c+2 c p+2 p(-5+8 p))} \\
& u_{3}=-\frac{\left(i_{1}-i_{2}\right)\left(i_{3}-i_{4}\right)\left(-i_{1}+i_{1}^{2}-i_{2}+i_{2}^{2}-2 i_{1} i_{2}+p-i_{1} p-i_{2} p\right)\left(-i_{3}+i_{3}^{2}-i_{4}+i_{4}^{2}-2 i_{3} i_{4}+p-i_{3} p-i_{4} p\right)}{2 p(p+1)(p+2)\left(c p+c+3 p^{2}-7 p+2\right)} .
\end{aligned}
$$

We find that $\chi_{i}=u_{i}$ where the central charge is set to $c=26-d$. Thus we see that the Virasoro symmetry emerges at the boundary of the constraints in (2.27), (2.28)!

Order $\boldsymbol{n}=4$. At $n=4$ a new phenomenon occurs. We find that by setting $\chi_{i}=u_{i}$ with $i=1,2,3$, there are no solutions for $\chi_{4}$ for which the equality in $(2.28)$ when applied to $\mathcal{C}_{0}^{(4)}$ is saturated for all positive external dimensions. To see this, let us simplify the problem to the vacuum block, $p=0$, and set $i_{1}=i_{2}=i, i_{3}=i_{4}=j$. In this case a general ansatz for $\chi_{4}$ is given as:

$$
\chi_{4}=\frac{g(i) g(j)}{a_{1} d^{2}+a_{2} d+a_{3}},
$$

where $g(i)$ is some polynomial. The boundary of the constraint in (2.28) becomes

$$
\mathcal{C}_{0}^{(4)}(i, i, i, i) \mathcal{C}_{0}^{(4)}(j, j, j, j)-\left(\mathcal{C}_{0}^{(4)}(i, i, j, j)\right)^{2}=0, \quad \forall i, j \geq 0 .
$$

With (2.38) the scalar coefficient takes the form

$$
\begin{aligned}
\mathcal{C}_{0}^{(4)}= & \frac{49\left(i^{2}+j^{2}\right)}{8\left(d^{2}-1\right)}+\frac{(7(5 d-142))(i+j)}{48\left(d^{2}-1\right)}+\frac{d(9 d-490)+6704}{384\left(d^{2}-1\right)}+\frac{189 i j(i+j)}{d^{3}-26 d^{2}-d+26} \\
& -\frac{(2(d+28)) i^{2} j^{2}}{d^{3}-26 d^{2}-d+26}+\frac{(d(d+680)-19166) i j}{30(d-26)(d-1)(d+1)}+\frac{g(i) g(j)}{a_{1} d^{2}+a_{2} d+a_{3}} .
\end{aligned}
$$

It is straightforward to see that there are no polynomial solutions for $g(i)$ that satisfy $(2.39)$. Thus there are no linear combinations of the global blocks that can saturate (2.28), so the true boundary is no longer given by the equality.

To seek the boundary, we begin with the following minimal ansatz:

$$
\chi_{4}=\frac{\left(i+a_{4} i^{2}\right)\left(j+a_{4} j^{2}\right)}{a_{1} d^{2}+a_{2} d+a_{3}} .
$$

For Virasoro block these parameters would be:

$$
a_{4}=5, a_{1}=\frac{25}{2}, a_{2}=-705, a_{3}=9880 .
$$

Now (2.39) becomes:

$$
\begin{aligned}
& \mathcal{C}_{0}^{(4)}(i, i, i, i) \mathcal{C}_{0}^{(4)}(j, j, j, j)-\left(\mathcal{C}_{0}^{(4)}(i, i, j, j)\right)^{2}= \\
& =\frac{(i-j)^{2}}{(d-26)^{2}(d-1)^{2}(d+1)}\left(f_{0}(j, d)+f_{1}(j, d) i+f_{2}(j, d) i^{2}\right)>0, \quad \forall i, j \geq 0
\end{aligned}
$$



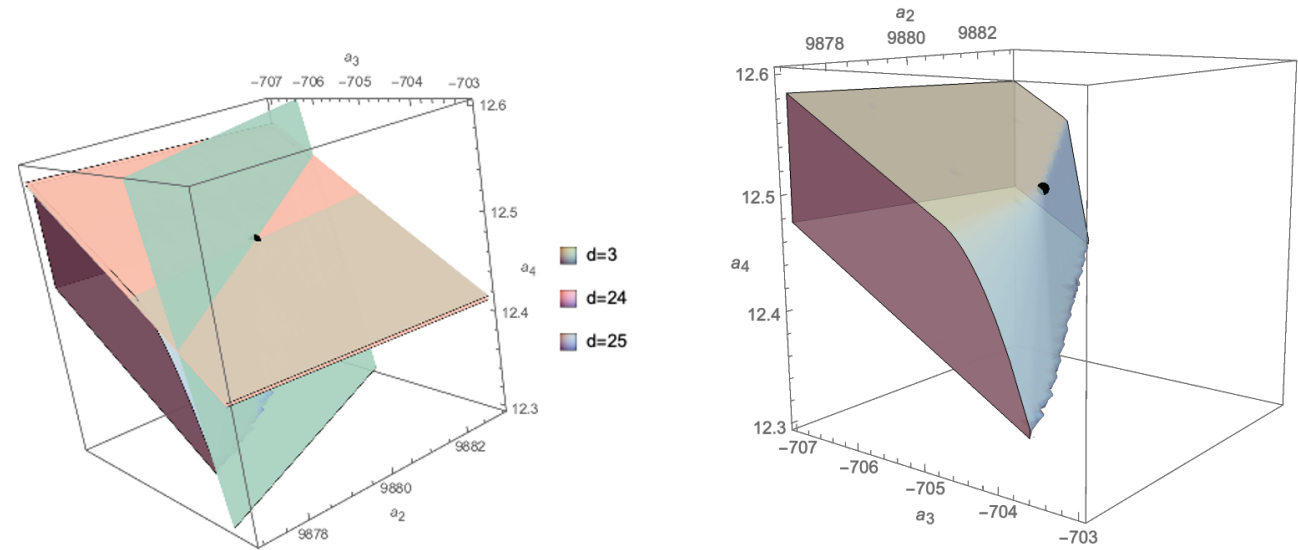

Figure 5. Solution space $\left(a_{1}, a_{2}, a_{3}\right)$ for ansatz (2.41). ( $a_{4}$ which we didn't plot here was fixed in $d=14$ positivity condition.) This diagram is carved out by inequalities $(2.45)$ corresponding to different dimensions, with $d=3,24,25$ shown here. In each dimension, the saturated inequality is a two-dimensional plane. The Virasoro point lives on the intersection of these planes.

where $f_{i}(j, d)$ 's are polynomial functions $j$ with maximal degree 2 . Since positivity rests on the sign of the last parenthesis, we can consider the space in $\left\{a_{i}\right\}$ carved out by the requirement that the polynomial inside can at most have a single root, so that it is never negative. ${ }^{5}$ For example in $d=14$, the discriminant of $i$ in the last parenthesis of (2.43) is:

$$
f_{1}(j, d)^{2}-4 f_{0}(j, d) f_{2}(j, d)=\left(a_{4}-5\right)^{2} \mathcal{F}\left(j, a_{1}, a_{2}, a_{3}, a_{4}\right) .
$$

Importantly, for any value of $\left\{a_{1}, a_{2}, a_{3}, a_{4}\right\}$ the function $\mathcal{F}$ will always have regions in $j$ for which it is positive, implying the existence of solutions for which (2.43) is negative. This tells us that $a_{4}=5$.

For the remaining coefficients, lets us see the image of $(2.43)$ in $\left(a_{1}, a_{2}, a_{3}\right)$ space. With $a_{4}=5,(2.43)$ yields the following inequalities:

$$
\begin{array}{ll}
d=3: & 0<9 a_{1}+3 a_{2}+a_{3} \leq 15755 / 2 \\
d=24: & 0<576 a_{1}+24 a_{2}+a_{3} \leq 160, \\
d=25: & 0<625 a_{1}+25 a_{2}+a_{3} \leq 135 / 2 .
\end{array}
$$

The carved out region is displayed in figure 5, where we have displayed the region as well as the respective hyperplane implied by the above inequalities. One can see from the figure that the Virasoro coefficients are set at a kink in the boundary. To make the last property manifest, let us consider a two-dimension sub-plane in figure 5 defined by $a_{1}=9880$, as shown in figure 6 . Then the Virasoro point is at the kink defined by the intersection of the constraints $d=3$ and 25 .

In summary, by considering the boundary carved out by the factorization conditions in (2.27) and (2.28), we find that the Virasoro block sits at unique special points on these boundaries.

\footnotetext{
${ }^{5}$ Of course for specific $i, j, \mathcal{C}_{0}^{(4)}(i, i, i, i) \mathcal{C}_{0}^{(4)}(j, j, j, j)-\left(\mathcal{C}_{0}^{(4)}(i, i, j, j)\right)^{2}$ may vanish. The previous discussion with regards to the boundary is about vanishing for all positive $(i, j)$.
} 


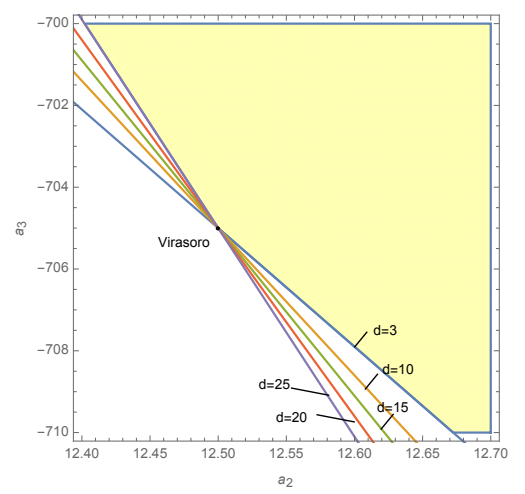

Figure 6. Solution space $\left(a_{2}, a_{3}\right)$ for ansatz (2.41) with $a_{1}$ fixed as Virasoro block $\left(a_{1}=9880\right)$. The lines denote the boundaries carved out by (2.11) (iii) in space-time dimension $d=3 \sim 25$. The point in the figure is the $a_{2}, a_{3}$ correspond to Virasoro block.

\section{Review of monodromy relations and the EFThedron}

\subsection{The monodromy plane}

The monodromy relation for amplitudes of massless external states:

$$
\mathcal{A}(2134)+e^{i \pi s} \mathcal{A}(1234)+e^{-i \pi u} \mathcal{A}(1324)=0,
$$

is a common feature shared by all flat-space open string amplitudes for identical external states. In fact when combined with the asymptotic form of the Regge limit, the above relation is sufficient to completely fix the four-point amplitude [30]. In particular, using the monodromy relations, one can fix the residue of anyone of the factorization channel to a single variable function with the real zeros fixed. If one further assumes that all residues match the form of the asymptotic Regge limit of the amplitude under $s \leftrightarrow t$ exchange, which for the Tachyon amplitude takes the form $t^{s+1}$ as $t \rightarrow \infty$, then this determines that there are no complex zeros and hence the residue is completely fixed up to an overall normalization. While it is not a priori clear that individual residues match the Regge asymptotics, we do expect that monodromy relations should imply stringent constraints when combined with unitarity.

The relation eq. (3.1) reflects the fact that the corresponding worldsheet integrand is permutation invariant, and it is only the ordering of the integration regions that characterizes the distinct orderings of the amplitude. ${ }^{6}$ For general string compactification, the monodromy around $z=0,1, \infty$, might deviate from the flat space counter-part. Assuming that the resulting monodromy is universal, i.e. it is simply an overall prefactor, the corresponding variation for (3.1) will be

$$
\mathcal{A}(2134)+e^{i \pi\left(s+a_{s}\right)} \mathcal{A}(1234)+e^{-i \pi\left(u+a_{u}\right)} \mathcal{A}(1324)=0 .
$$

\footnotetext{
${ }^{6}$ For example the Lovelace-Shapiro amplitude:

$$
\mathcal{A}^{(L S)}(1234)=g^{2} \frac{\Gamma\left(\frac{1}{2}-s\right) \Gamma\left(\frac{1}{2}-t\right)}{\Gamma(-s-t)}
$$

will not satisfy the above monodromy relations since the external states are not identical [41].
} 
Note that the monodromy relation, along with two other ones from permutations $1 \leftrightarrow 2$ and $1 \leftrightarrow 3$, result in 6 real conditions. Since we only have three independent amplitudes, $A(1234), A(2134)$, and $A(1324),{ }^{7}$ in order for there to be a non-trivial solution we can only have $a_{s}=a_{t}=a_{u}=0, \frac{2}{3}, \frac{4}{3}$. To see this, we note that for there to be a solution the 6 linear constraints from 3 different monodromy relations must degenerate to at most 2 . Therefore there can be only one independent monodromy relation. In other words, the three different monodromy relations must be proportional to each other and this proportionality requirement fixes $a_{s}=a_{t}=a_{u}=0, \frac{2}{3}, \frac{4}{3}$.

It is instructive to see how such deformed monodromy arrises upon compactification of flat space string amplitudes. Consider the compactification of the Tachyon amplitude in bosonic string theory, with the 26 dimensional momenta denoted as $K_{i}$ (with $\alpha^{\prime} K_{i}^{2}=1$ ).

$$
A^{(26 D)}(1234)=\frac{1}{\mathrm{Vol}} \int \prod_{i=1}^{4} d z_{i} \prod_{j<i=2}^{4}\left(z_{i}-z_{j}\right)^{2 \alpha^{\prime} K_{i} \cdot K_{j}} .
$$

By decomposing the momenta into $d$ and $26-d$ components, $K_{i}=\left(k_{i}, q_{i}\right)$ with $k_{i}^{2}=0$, we obtain the compactified $d$-dimensional amplitude as:

$$
A^{(4 D)}(1234)=\frac{1}{\mathrm{Vol}} \int \prod_{i=1}^{4} d z_{i} \prod_{j<i=2}^{4}\left(\left(z_{i}-z_{j}\right)^{2 \alpha^{\prime} k_{i} \cdot k_{j}}\left(z_{i}-z_{j}\right)^{2 \alpha^{\prime} q_{i} \cdot q_{j}}\right) .
$$

Note that the above is permutation invariant under $\left\{z_{i}, k_{i}\right\} \leftrightarrow\left\{z_{j}, k_{j}\right\}$ only if all $q_{i} \cdot q_{j}$ 's are equal. The mass shell condition

$$
2 \alpha^{\prime}\left(q_{1} \cdot q_{2}+q_{1} \cdot q_{3}+q_{1} \cdot q_{4}\right)=-2 \alpha^{\prime}\left(k_{1}^{2}-K_{1}^{2}\right)=2,
$$

then fixes $2 \alpha^{\prime} q_{i} \cdot q_{j}$ to $\frac{2}{3}$, which leads to an additional phase of $\frac{2}{3} \pi$ or $\frac{4}{3} \pi$. Such "twisted monodromy relations" (3.2) result in amplitudes without massless poles. Indeed expanding in $\alpha^{\prime}$, the leading order identity is:

$$
A_{\mathrm{IR}}(2134)+e^{2 i \pi / 3} A_{\mathrm{IR}}(1234)+e^{4 i \pi / 3} A_{\mathrm{IR}}(1324)=0,
$$

which indicates that the leading order amplitude $A_{I R}$ can only be a constant. While such amplitudes are potentially interesting objects to study, we will be focusing on four-point amplitudes where massless poles are present, thus we will restrict ourselves to the standard monodromy relation (3.1). Generalizing to the twisted case is straightforward.

The monodromy relation (3.1) imposes restrictive constraints on the amplitude. First of all, it implies integer spectrum of the theory, which is evident by looking at the imaginary part of the four-point monodromy relation $\sin (\pi s) \mathcal{A}(1234)=\sin (\pi u) \mathcal{A}(1324)$ The $s$ channel and $u$-channel poles must be paired with the zeros in the respective sine factors for this identity to hold for all values of $s, t, u$, while all zeros of the sine function are located at integer values. Although very powerful, we will not make use of this fact, which is left to future studies. More importantly for us, the monodromy relations imply nontrivial

\footnotetext{
${ }^{7}$ With the cyclic invariance $A(1234)=A(4123)$ and reflection symmetry $A(1234)=A(4321)$ any four-point amplitude with arbitrary order of $1,2,3,4$ can be identified with one of the three.
} 
mixing relations of couplings at different derivative order, which will be the focus of this subsection. Note that in the low energy limit, these relations turn into the field theory Kleiss-Kuijif and Bern-Carrasco-Johansson relations [42, 43].

We will be interested in the case where the complete amplitude is proportional to the leading order in $\alpha^{\prime}$ expansion,

$$
\mathcal{A}(1234)=S\left(\left\{k_{i}, \epsilon_{i}\right\}\right) A(s, t)=S\left(\left\{k_{i}, \epsilon_{i}\right\}\right)\left(-\frac{1}{s t}+\cdots\right),
$$

where $S$ is the universal common factor that is independent of the ordering. This occurs when we have four-dimensional massless external states, where the prefactor is completely fixed by the helicity weights, or maximal supersymmetry in general dimensions, where the external states are in the same multiplet. The monodromy relation (3.1) then becomes

$$
A(s, u)+e^{i \pi s} A(s, t)+e^{-i \pi u} A(t, u)=0,
$$

and the constraint at each derivative order can be explicitly written in terms of Laurent coefficients of $A(s, t)$. To study the constraint we first write the factor $A(s, t)$ in (3.7) as an expansion

$$
A(s, t)=-\frac{1}{s t}+\left(\frac{b}{s}+\frac{b}{t}\right)+\left(c \frac{t}{s}+c \frac{s}{t}\right)+g_{00}+\left(g_{1,0} s+g_{1,1} t\right)+\sum_{k \geq q \geq 0} g_{k, q} s^{k-q} t^{q},
$$

then solve for the monodromy constraints imposed by (3.8) order by order in $\alpha^{\prime}$. First, one immediately finds the coefficient for $t^{2 n} / s$ must be zero, including $b=0$. For $c$, as we will demonstrate in the next section, the unitarity constraint will also set it to zero. Thus for the remainder of the discussion we will solve the monodromy relations with respect to

$$
A(s, t)=-\frac{1}{s t}+\sum_{k \geq q \geq 0} g_{k, q} s^{k-q} t^{q} .
$$

As an example, the solution up to $k=4$ is given as:

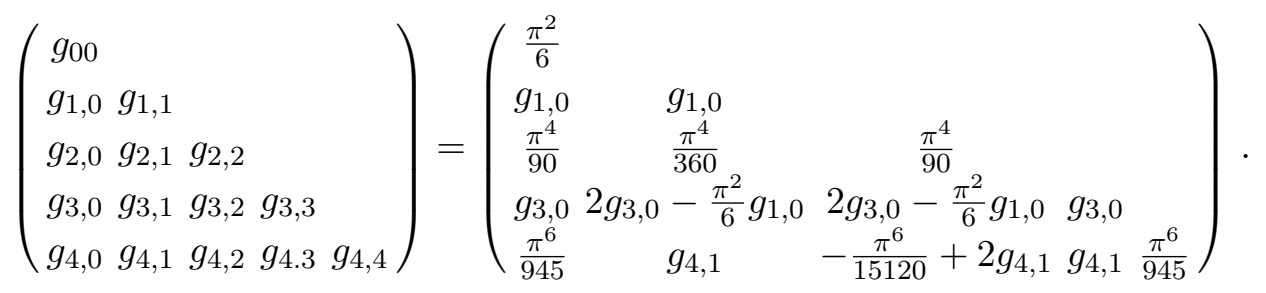

We will refer to the above solution as the monodromy plane, defining a subspace in the EFT couplings where the monodromy conditions are satisfied order by order. Our next step will be to constrain the remaining parameters with positivity conditions. But first, let us compare our solution with the actual superstring $A(s, t)$ factor, given by [44]

$$
A_{\text {Superstring }}(s, t)=\frac{\Gamma(-s) \Gamma(-t)}{\Gamma(1-s-t)}=\frac{1}{s t} \exp \left(\sum_{n \geq 2} \frac{\zeta(n)\left((-s)^{n}+(-t)^{n}-(-s-t)^{n}\right)}{n}\right) .
$$


We can confirm that all fixed $g_{k, q}$ coefficients in (3.11) match with the string value after expressing the even zeta values, $\zeta(2)=\frac{\pi^{2}}{6}, \zeta(4)=\frac{\pi^{4}}{90}$, and so on. In particular, it will be useful later on to observe that the coefficients $g_{2 n, 0}$ are all set to $\zeta(2 n)$ by monodromy. But we also observe a correspondence between the remaining independent coefficients and the monomials of odd zeta values, a pattern that also persists to higher orders, as the string value of the free parameters on the monodromy plane reads:

$$
g_{1,0}=\zeta(3), \quad g_{3,0}=\zeta(5), \quad g_{4,1}=\frac{\pi^{6}}{1260}-\frac{1}{2} \zeta^{2}(3) .
$$

This is closely related to the fact that only certain linear combinations of the remaining free parameters survive double copy (we will show the explicit formula in (4.26)), and the closed superstring amplitude only contains odd zeta values [45, 46].

As we will see, the EFThedron constraints will be able to fix the remaining parameters to (3.13) with high precision.

\subsection{The EFThedron}

The unitarity constraint on factorization also leaves its fingerprint on the low energy amplitude, in the form of positivity bounds on the EFT couplings. A precursor of such bounds is the positivity of the leading four derivative operator derived from optical theorem in [20]. More recently, an infinite set of positivity bounds have been derived by considering the near forward limit of the low energy expansion, exploiting the positive geometry that arises from expanding the Gegenbauer polynomials [25]. Here we present a brief review.

Let us consider the low energy limit of an UV complete amplitude. Here, by low energy we are referring to the limit where the Mandlestam variables are smaller than the scale set by the UV massive states. For an ordered amplitude, in this limit the amplitude takes the form:

$$
\left.A(s, t)\right|_{s, t \ll 1}=\frac{a}{s t}+\frac{b_{1}}{s}+\frac{b_{2}}{t}+\sum_{k, q \geq 0} g_{k, q} s^{k-q} t^{q},
$$

where we have set the UV scale to 1 , and $a, b_{1}, b_{2}$ can be some kinematic dependent functions. Cyclic symmetry means that we can identify $g_{k, q}=g_{k, k-q}$. Note that we have defined the EFT couplings $g_{k, q}$ from the polynomial expansion of the low energy amplitude. This allows us to define the couplings via a contour integral in the complex $s$-plane, with $t$ held fixed:

$$
g_{k, q}=\left.\frac{1}{q !} \frac{d^{q}}{d t^{q}}\left(\frac{i}{2 p} \oint \frac{d s}{s^{k-q+1}} A(s, t)\right)\right|_{t=0} .
$$

By deforming the contour, one picks up the residues and discontinuity on the real positive $s$-axes (since we do not have $u$-channel thresholds), arising from

$$
\left.A(s, t)\right|_{s \rightarrow m^{2}}=\sum_{\ell} c_{\ell} \frac{G_{\ell}^{d}(\cos \theta)}{s-m^{2}}, \quad \cos \theta=1+\frac{2 t}{m^{2}},
$$

where $c_{\ell} \geq 0$. While the above form describes the behavior of the amplitude near treethreshold, near the forward limit the same form holds for branch cuts, except that one has to sum over a continuous spectrum. 
In other words, combining (3.15) with (3.16), we have that the low energy coupling can be matched to the derivative expansion of the Gegenbauer polynomials as well as the propagators. Defining the Taylor coefficients $v_{\ell, q}$ from:

$$
G_{\ell}^{d}(1+\delta)=\sum_{q=0} v_{\ell, q} \delta^{q}
$$

at fixed $k$, fixed mass dimension of the operator, we find:

$$
\vec{g}_{k}=\sum\left(\begin{array}{c}
g_{k, 0} \\
g_{k, 1} \\
\vdots \\
g_{k, n}
\end{array}\right) \in \sum_{a} c_{a} \overrightarrow{\mathcal{G}}_{\ell_{a}}, \quad \overrightarrow{\mathcal{G}}_{\ell}=\left(\begin{array}{c}
v_{\ell, 0} \\
v_{\ell, 1} \\
\vdots \\
v_{\ell, n}
\end{array}\right),
$$

where $a$ labels the spectrum of the UV states and $c_{a}>0$. The above implies the $n+1$ component vector $\vec{g}_{k}$ must lie inside the convex hull of the Gegenbauer vectors $\overrightarrow{\mathcal{G}}_{\ell}$. Importantly, due to the positivity properties of $\overrightarrow{\mathcal{G}_{\ell}}$, its convex hull is a cyclic polytope, and the boundary of the hull is constructed by adjacent pairs of $\overrightarrow{\mathcal{G}}_{\ell}$. For example for $n=4, \vec{g}_{k}$ being inside the convex hull implies that:

$$
\left\langle\vec{g}_{k}, \ell_{i}, \ell_{i}+1, \ell_{j}, \ell_{j}+1\right\rangle=\operatorname{det}\left[\vec{g}_{k}, \overrightarrow{\mathcal{G}}_{\ell_{i}}, \overrightarrow{\mathcal{G}}_{\ell_{i}+1}, \overrightarrow{\mathcal{G}}_{\ell_{j}}, \overrightarrow{\mathcal{G}}_{\ell_{j}+1}\right] \equiv \vec{g}_{k} \cdot W_{I} \geq 0
$$

where we use $W_{I}$ as a short hand notation for the boundary, here given by $\left\langle *, \ell_{i}, \ell_{i}+1, \ell_{j}\right.$, $\left.\ell_{j}+1\right\rangle$. Note that since for any vector $\vec{g}_{k}$ that satisfies the above constraint, rescaling by a positive constant yields another solution, the geometry of the convex hull is really a polytope in $\mathbb{P}^{n}$. For $n=$ odd, we have the same pattern for the boundaries except with an extra vector $\overrightarrow{\mathcal{G}_{0}}$.

We can also consider keeping $q$ fixed while collecting the couplings with distinct $k$. We then have:

$$
\vec{g}_{q}=\sum\left(\begin{array}{c}
g_{q, q} \\
g_{q+1, q} \\
\vdots \\
g_{q+n, q}
\end{array}\right) \in \sum_{a} c_{a} v_{q}\left(x_{a}\right)^{q}\left(\begin{array}{c}
1 \\
x_{a} \\
\vdots \\
x_{a}^{n}
\end{array}\right) \quad x_{a}=\frac{1}{m_{a}^{2}} .
$$

That is, the vector $\vec{g}_{q}$ lives in the convex hull of points on a moment curve, $\left(1, x, x^{2}, \cdots, x^{n}\right)$. This is a reflection that fixed $q$ means we are expanding the Gegenbauer polynomial to fixed order, and collecting the expansion of $1 /\left(s-m^{2}\right)$ which gives a geometric series. Importantly, since we have $x_{a}>0, \vec{g}_{q}$ really lives in the convex hull of a half moment curve $\left(1, x, x^{2}, \cdots, x^{n}\right)$ with $x \in R^{+}$. This implies that the Hankel matrix for the couplings, defined as the following symmetric matrix:

$$
H=\left(\begin{array}{cccc}
g_{k, q} & g_{k+1, q} & \cdots & g_{k+n, q} \\
g_{k+1, q} & g_{k+2, q} & \cdots & g_{k+1+n, q} \\
\vdots & \vdots & \ddots & \vdots \\
g_{k+1+n, q} & g_{k+2+n, q} & \cdots & g_{k+2 n, q}
\end{array}\right),
$$

will have all non-negative minors. 
Finally, the fact that the expansion in $s$ and $t$ corresponds to a direct product of geometries is reflected in the following product Hankel matrix constraint:

$$
H=\left(\begin{array}{cccc}
\vec{g}_{k} \cdot W_{I} & \vec{g}_{k+1} \cdot W_{I} & \cdots & \vec{g}_{k+1} \cdot W_{I} \\
\vec{g}_{k+1} \cdot W_{I} & \vec{g}_{k+2} \cdot W_{I} & \cdots & \vec{g}_{k+1+n} \cdot W_{I} \\
\vdots & \vdots & \ddots & \vdots \\
\vec{g}_{k+1+n} \cdot W_{I} & \vec{g}_{k+2+n} \cdot W_{I} & \cdots & \vec{g}_{k+2 n} \cdot W_{I}
\end{array}\right),
$$

where $W_{I}$ is any one of the cyclic polytope boundaries in $\mathbb{P}^{n}$.

The space of couplings carved out by the above inequalities is termed the EFThedron [25], which we briefly summarize:

- Cyclic polytope constraints:

$$
\mathrm{n} \in \text { even }:\left\langle\vec{g}_{k}, \ell_{i}, \ell_{i}+1, \ell_{j}, \ell_{j}+1, \cdots\right\rangle \geq 0, \quad \mathrm{n} \in \text { odd }:\left\langle\mathbf{0}, \vec{g}_{k}, \ell_{i}, \ell_{i}+1, \ell_{j}, \ell_{j}+1, \cdots\right\rangle \geq 0
$$

where $\mathbf{0}$ represents $\overrightarrow{\mathcal{G}_{0}}$.

- Hankel matrix constraints:

$$
\operatorname{Minor}\left[\begin{array}{cccc}
g_{k, q} & g_{k+1, q} & \cdots & g_{k+n, q} \\
g_{k+1, q} & g_{k+2, q} & \cdots & g_{k+1+n, q} \\
\vdots & \vdots & \vdots & \vdots \\
g_{k+1+n, q} & g_{k+2+n, q} & \cdots & g_{k+2 n, q}
\end{array}\right] \geq 0
$$

- Product Hankel matrix constraints:

$$
\text { Minor }\left[\begin{array}{cccc}
\vec{g}_{k} \cdot W_{I} & \vec{g}_{k+1} \cdot W_{I} & \cdots & \vec{g}_{k+1} \cdot W_{I} \\
\vec{g}_{k+1} \cdot W_{I} & \vec{g}_{k+2} \cdot W_{I} & \cdots & \vec{g}_{k+1+n} \cdot W_{I} \\
\vdots & \vdots & \vdots & \vdots \\
\vec{g}_{k+1+n} \cdot W_{I} & \vec{g}_{k+2+n} \cdot W_{I} & \cdots & \vec{g}_{k+2 n} \cdot W_{I}
\end{array}\right] \geq 0 .
$$

\section{Intersection of monodromy plane and the EFThedron}

In this section, we investigate the allowed space of Laurent coefficients under the combined constraints following from the monodromy relation (3.8) and unitarity in section 3.2 for four-point amplitudes in four-dimensional space-time. First we demonstrate that monodromy and positivity of Hankel matrices rule out the $t / s$ and $s / t$ poles that correspond to vector exchange. Note that since the EFT couplings are defined with an overall prefactor in eq. (3.7), which behaves as $s^{2}$, the Froissart bound implies that the EFT couplings $g_{k, q}$ are subject to EFThedron constraints for $k, q \geq 0 .{ }^{8}$ Next, we apply the full EFThedron constraints on the remaining monodromy plane of the open and closed string EFT amplitudes.

\footnotetext{
${ }^{8}$ More precisely the Froisart bound is applicable to the subtracted amplitude, defined as $\mathcal{A}_{\text {sub }}=\mathcal{A}+$ $\frac{S\left(\left\{k_{i}, \epsilon_{i}\right\}\right)}{s t}$. Both $\mathcal{A}_{\text {sub }}$ and $\mathcal{A}$ share the same EFT expansion.
} 


\subsection{The absence of isolated massless poles}

In section 3.1 it was stated that monodromy relations, when combined with unitarity, enforce the massless pole structure of $A(s, t)$ to be only of the form $\frac{1}{s t}$. Here we give the derivation. Starting with the following Laurent expansion of the four-point amplitude:

$$
A(s, t)=-\frac{1}{s t}+c\left(\frac{t}{s}+\frac{s}{t}\right)+\frac{b}{s}+\frac{b}{t}+\sum_{k \geq q \geq 0} g_{k, q} s^{k-q} t^{q} .
$$

We first impose the monodromy relation to the above, leading to the following solutions for the couplings up to $k=4$ :

$$
\begin{array}{rlrl}
k & =-1: & b & =0 \\
k=0: & g_{0,0} & =\zeta(2)+c \\
k=2: & g_{2,0} & =g_{2,2}=\zeta(4)-\zeta(2) c \\
& g_{2,1} & =\frac{1}{4} \zeta(4)-\zeta(2) c \\
k=3: & g_{3,1} & =g_{3,2}=2 g_{3,0}-\zeta(2) g_{1,0} \\
k & =4: & g_{4,0} & =g_{4,4}=\zeta(6)-\zeta(4) c \\
g_{4,2} & =-\frac{1}{16} \zeta(6)+\frac{1}{4} \zeta(4) c+2 g_{4,1} .
\end{array}
$$

Once again one sees that $b$ is set to zero by monodromy relations alone. Furthermore, $g_{2 n, 0}$ is solved in terms of $c$ alone and has the following general form:

$$
g_{2 n, 0}=\zeta(2 n+2)-\zeta(2 n) c .
$$

We consider the minor of Hankel matrix (3.24):

$$
H_{1, N \times N}=\left(\begin{array}{cccc}
g_{0,0} & g_{2,0} & \cdots & g_{2 N-2,0} \\
g_{2,0} & g_{4,0} & \cdots & g_{2 N, 0} \\
\vdots & \vdots & \ddots & \vdots \\
g_{2 N-2,0} & g_{2 N, 0} & \cdots & g_{4 N-4,0}
\end{array}\right), \quad H_{2, N \times N}=\left(\begin{array}{cccc}
g_{2,0} & g_{4,0} & \cdots & g_{2 N, 0} \\
g_{4,0} & g_{6,0} & \cdots & g_{2 N+2,0} \\
\vdots & \vdots & \ddots & \vdots \\
g_{2 N, 0} & g_{2 N+2,0} & \cdots & g_{4 N-2,0}
\end{array}\right)
$$

The positive condition $\operatorname{det}\left(H_{i}\right)>0$ up to $N=30$ implies the condition $-4.24 \times 10^{-6}<$ $c<6.81 \times 10^{-6}$, so we can conclude $c$ is asymptotically fixed to zero.

Thus we conclude that we can simply begin with

$$
A(s, t)=-\frac{1}{s t}+\sum_{k \geq q \geq 0} g_{k, q} s^{k-q} t^{q},
$$

and study the intersection geometry further.

\subsection{Combined constraints}

In section 3.2 we showed that unitarity implies two different types of constraints: being inside the cyclic polytope (3.23), and the positivity of Hankel matrices (3.24), (3.25), which 
we already used in ruling out the $\frac{s}{t}$ and $\frac{t}{s}$ terms. In the parameter space for all independent $g_{k, q}$, these unitary constraints carve out the EFThedron, a positive region bounded by a set of codimension one surfaces. On the other hand, the monodromy constraint fixes a subset of $g_{k, q}$ and imposes linear relations among the unfixed ones, therefore defines a lower dimensional plane, which we call the monodromy plane. The allowed Laurent coefficients must lie in the intersection of the EFThedron and the monodromy plane. The final shape of the allowed region can be straightforwardly derived by imposing (3.23), (3.24) and (3.25) on this hyperplane.

By setting $c=0$ in (4.2), we arrive at the following defining relations for the monodromy plane:

$$
\begin{array}{ll}
k=0: & g_{0,0}=\zeta(2) \\
k=2: & g_{2,0}=g_{2,2}=\zeta(4) \\
& g_{2,1}=\zeta(4) / 4 \\
k=3: & g_{3,1}=g_{3,2}=2 g_{3,0}-\zeta(2) g_{1,0} \\
k=4: & g_{4,0}=g_{4,4}=\zeta(6) \\
& g_{4,2}=-\zeta(6) / 16+2 g_{4,1},
\end{array}
$$

where we choose $g_{1,0}$ and $g_{3,0}$ as the free parameters for the hyperplane with $k \leq 3$, and $g_{1,0}$, $g_{3,0}$, and $g_{4,1}$ for $k \leq 4$. In the rest of this section, most of our analysis for constraints will be carried out in this two-dimensional reduced space for $\left(g_{1,0}, g_{3,0}\right)$ and three-dimensional reduced space for $\left(g_{1,0}, g_{3,0}, g_{4,1}\right)$. We will derive and illustrate graphically the region carved out by increasing order of unitarity constraints and show how the results leads to our main conjecture. The carving process starts with low order unitarity constraints ( $k=3,4$ respectively for the two and three-dimensional reduced space), in which case all unitarity constraints are in terms of the reduced space coordinates and can be fully solved analytically. We then further carve the region by including higher order unitary constraints, which often involve higher order monodromy free parameters $g_{5, q}, g_{6, q}, g_{7, q}$, etc. Our numerical investigation shows that higher order constraints indeed carve out an increasingly smaller region within the lower order unitarity region.

Note that the space-time dimension needs to be specified at the beginning of the carving procedure, as the result is dimensional dependent due to the dimensional dependence of the cyclic polytope (3.23) and product Hankel matrices (3.25). We will focus on amplitudes in four-dimensional space-time, while the process can be easily replicated for amplitudes in other dimensions. In general, the carved out region for higher dimensional amplitudes will lie within the four-dimensional result.

\subsubsection{The $k=3$ geometry}

We will start by considering the unitarity constraints only up to the order $k=3$. First we consider the cyclic polytope constraint for $k=2$, which will bound $\vec{g}_{2}=$ $\left(1, g_{2,1} / g_{2,0}, g_{2,2} / g_{2,0}\right)$. From cyclic symmetry we have $g_{2,1}=g_{2,2}$, and so $\vec{g}_{2}$ is one parameter vector with 3 components. We have the strongest condition from: $\left\langle\overrightarrow{g_{2}} \overrightarrow{\mathcal{G}_{1}} \overrightarrow{\mathcal{G}_{2}}\right\rangle>0$. 
The vector $\overrightarrow{\mathcal{G}}_{\ell}$ of the four-dimensional Gegenbauer polynomial $G_{\ell}^{1 / 2}(\cos \theta)$ reads:

$$
\overrightarrow{\mathcal{G}_{1}}=(1,2,0), \quad \overrightarrow{\mathcal{G}_{2}}=(1,6,6) .
$$

For $k=3, \vec{g}_{3}=\left(1, g_{3,1} / g_{3,0}, g_{3,2} / g_{3,0}, g_{3,3} / g_{3,0}\right)$ is still a one parameter vector, with 4 components. The strongest condition is given by considering the cyclic polytope in one lower dimension, which means we only keep components $g_{3, q}$ from $q=0$ to 2 . We use prime to denote this vector $\vec{g}_{3}^{\prime}=\left(1, g_{3,1} / g_{3,0}, g_{3,2} / g_{3,0}\right)$ and the extremal condition is: $\left\langle\vec{g}_{3}^{\prime} \overrightarrow{\mathcal{G}_{1}} \overrightarrow{\mathcal{G}_{2}}\right\rangle>0$. As a result, the two cyclic polytope constraints are as follows

$$
0 \leq \frac{g_{2,1}}{g_{2,0}} \leq \frac{8}{3}, \quad 0 \leq \frac{g_{3,1}}{g_{3,0}} \leq 6, .
$$

The Hankel matrix constraints up to order $k=3$ consist of the positivity of all $g_{k, q}$, as well as positivity of the determinants of the following three matrices:

$$
H_{1}=\left(\begin{array}{ll}
g_{0,0} & g_{1,0} \\
g_{1,0} & g_{2,0}
\end{array}\right), H_{2}=\left(\begin{array}{ll}
g_{1,0} & g_{2,0} \\
g_{2,0} & g_{3,0}
\end{array}\right), H_{3}=\left(\begin{array}{ll}
g_{1,1} & g_{2,1} \\
g_{2,1} & g_{3,1}
\end{array}\right),
$$

which reduce to the following constraints on the Laurent coefficients

$$
\begin{aligned}
& \operatorname{det}\left(H_{1}\right)=g_{2,0} g_{0,0}-g_{1,0}^{2}>0, \\
& \operatorname{det}\left(H_{2}\right)=g_{1,0} g_{3,0}-g_{2,0}^{2}>0, \\
& \operatorname{det}\left(H_{3}\right)=g_{1,0} g_{3,1}-g_{2,1}^{2}>0 .
\end{aligned}
$$

In the $g_{k, q}$ space of parameters, the monodromy plane is defined by the linear relations in (4.6). In the subspace of $g_{k, q}$ for $k \leq 3$, the monodromy plane is a two-dimensional plane parameterized by coordinates $\left(x=g_{1,0}, y=g_{3,0}\right)$, with explicit coordinate representation:

$$
\left(g_{0,0}, g_{1,0}, g_{2,0}, g_{2,1}, g_{3,0}, g_{3,1}\right)=(\zeta(2), x, \zeta(4), \zeta(4) / 4, y, 2 y-\zeta(2) x) .
$$

On this plane, positivity of $g_{1,0}$ and $g_{3,0}$ translates to positivity of coordinates, and the space-time dimensional independent part of cyclic polytope constraints (4.8) reduces to a linear inequality

$$
y>\frac{\zeta(2)}{2} x,
$$

while the dimensional dependent part is trivial, for positive $x$ and $y$. Finally, the positive Hankel matrix determinants in (4.10) reduce to the following quadratic constraints on $x$ and $y$ :

$$
\begin{aligned}
& \operatorname{det}\left(H_{1}\right)=-x^{2}+\zeta(4) \zeta(2)>0, \\
& \operatorname{det}\left(H_{2}\right)=x y-\zeta(4)^{2}>0, \\
& \operatorname{det}\left(H_{3}\right)=-\zeta(2) x^{2}+2 x y-\frac{1}{16} \zeta(4)^{2}>0 .
\end{aligned}
$$

The constraints are demonstrated graphically in figure $7 \mathrm{a}$, and their intersection is magnified in figure $7 \mathrm{~b}$, along with a marked point corresponding to the open string solution of $(x, y)$. 


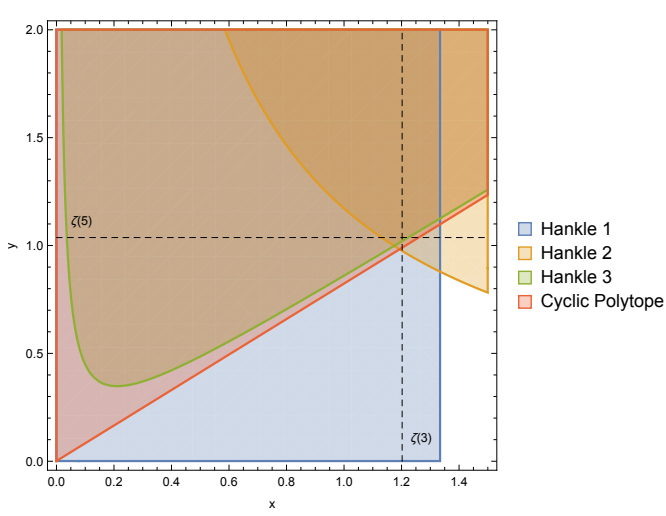

(a) Hankel matrix and cyclic polytope constraints up to $k=3$.

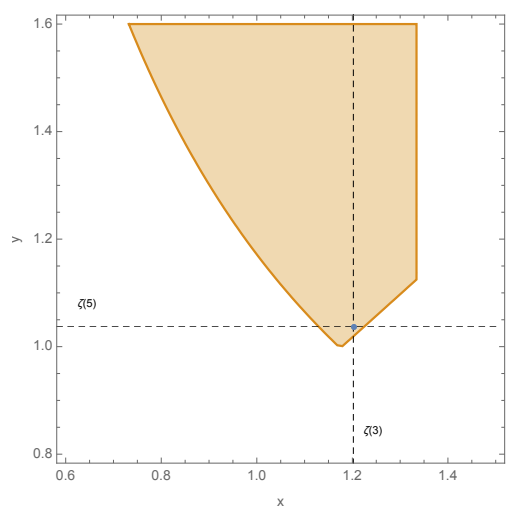

(b) The region of intersection and position of superstring solution.

Figure 7. Region of parameter space carved out by monodromy and EFT plane up to $k=3$.

\subsubsection{The $k=4$ geometry}

Moving on to $k=4$ geometry, we demonstrate the crucial feature that at fixed $k$, including Hankel matrices (3.24) constraints of higher order $k$ not only bounds the new degrees of freedom, it further constrains the low order parameters. For instance, as monodromy requires $g_{4,0}=\zeta(6)$, we can improve the Hankel matrix constraints for $\left\{g_{k, q} \mid k \leq 3\right\}$ by including the Hankel matrices involving $\left\{g_{4,0}\right\}$

$$
H_{4}=\left(\begin{array}{lll}
g_{0,0} & g_{1,0} & g_{2,0} \\
g_{1,0} & g_{2,0} & g_{3,0} \\
g_{2,0} & g_{3,0} & g_{4,0}
\end{array}\right), \quad H_{5}=\left(\begin{array}{ll}
g_{2,0} & g_{3,0} \\
g_{3,0} & g_{4,0}
\end{array}\right), \quad H_{6}=\left(\begin{array}{ll}
g_{0,0} & g_{2,0} \\
g_{2,0} & g_{4,0}
\end{array}\right)
$$

which leads to two new constraints on the monodromy plane (4.11):

$$
\operatorname{det}\left(H_{4}\right)=-\frac{4}{7} \zeta(4) x^{2}+\frac{4}{5} \zeta(2) x y-y^{2}+\frac{3}{25} \zeta(2)^{2} \zeta(6)>0,
$$

and

$$
\operatorname{det}\left(H_{5}\right)=\zeta(4) \zeta(6)-y^{2}>0 .
$$

Other constraints at order $k=4$ arise from the polytope constraint, Hankel matrices containing order 4 parameter $g_{4,1}$, and the product Hankel matrices. The polytope constraint (3.23) at $k=4$ reads

$$
\left\langle\vec{g}_{4} \overrightarrow{\mathcal{G}}_{0} \overrightarrow{\mathcal{G}}_{i} \overrightarrow{\mathcal{G}}_{i+1} \overrightarrow{\mathcal{G}}_{j} \overrightarrow{\mathcal{G}}_{j+1}\right\rangle>0, \quad \vec{g}_{4}=\left(1, g_{4,1} / g_{4,0}, g_{4,2} / g_{4,0}, g_{4,3} / g_{4,0}, g_{4,4} / g_{4,0}\right),
$$

where $\vec{g}_{4}$ only has two independent components due to the symmetry $g_{k, q}=g_{k, k-q}$. Eq. (4.17) gives rise to more than two hundred inequalities for the variables $\left(g_{1,0}, g_{3,0}, g_{4,1}\right)$, but their intersection simplifies to the following inequality of $g_{4,1}$

$$
\frac{\pi^{6}}{30240} \leq g_{4,1} \leq \frac{\pi^{6}}{1512},
$$


which is compatible with any value of $(x, y)=\left(g_{1,0}, g_{3,0}\right)$ and imposes no further constraints on $(x, y)$-plane.

The following two Hankel matrices containing $g_{4,1}$ also lead to nontrivial constraints

$$
H_{7}=\left(\begin{array}{ll}
g_{2,1} & g_{3,1} \\
g_{3,1} & g_{4,1}
\end{array}\right), \quad H_{8}=\left(\begin{array}{ll}
g_{2,2} & g_{3,2} \\
g_{3,2} & g_{4,2}
\end{array}\right)
$$

In terms of $(x, y, z)$, their positive determinants read

$$
\begin{array}{ll}
\operatorname{det}\left(H_{7}\right)>0: & \frac{\zeta(4)}{4} z-(2 y-\zeta(2) x)^{2}>0 \\
\operatorname{det}\left(H_{8}\right)>0: & \zeta(4)\left(2 z-\frac{\zeta(6)}{16}\right)-(2 y-\zeta(2) x)^{2}>0 .
\end{array}
$$

Which again compatible with all values for $(x, y)$.

Comparing to the $k=3$ case, starting at $k=4$, there are new types of constraints (3.25) arising from product Hankel matrices. At $k=4$, there are ten different product Hankel matrices, whose specific form depends on space-time dimension. Here we only list two of the ten such product Hankel matrices as examples

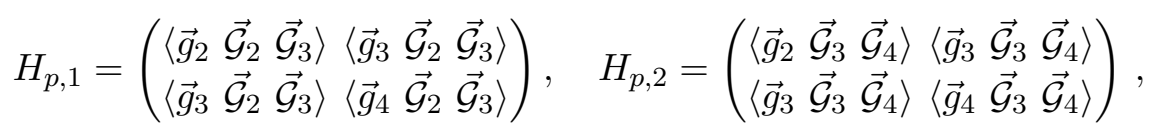

implying the following conditions:

$$
\begin{aligned}
& \operatorname{det}\left(H_{p, 1}\right)=41 \pi^{6}-75 \pi^{4} x^{2}-43200 y^{2}-720 \pi^{2}(5 x y+z)>0, \\
& \operatorname{det}\left(H_{p, 2}\right)=64801 \pi^{6}-60\left(845 \pi^{4} x^{2}+1590480 y^{2}+3 \pi^{2}(24440 x y+5203 z)\right)>0 .
\end{aligned}
$$

Eqs. (4.20) and (4.22) are constraints involving parameters $(x, y, z)$, so the region they carve needs to be projected to the two-dimensional plane of $(x, y)$. It turns out the projections of the $k=4$ constraints (4.18), (4.20) and (4.22) are either trivial or weaker than previous constraints. After reduction the intersection of all possible constraints up to $k=4$ simplifies to the intersection of (4.13) and (4.15), which is shown in figure 8a and magnified in figure $8 \mathrm{~b}$. As manifested by figure $8 \mathrm{c}$, the $k=4$ allowed region for $x, y$ is notably smaller than the $k=3$ one.

At this stage, $k=4$, we are able to fix the coordinates $x$ and $y$ on the monodromy plane to within $10 \%$ accuracy around the actual values of $\zeta(3)=1.20 \ldots$ and $\zeta(5)=1.03 \ldots$ respectively. Moreover, the string value appears to be sitting very close the boundary of the new allowed region. ${ }^{9}$

We now return to the three-dimensional monodromy plane defined by $(x, y, z)=$ $\left(g_{1,0}, g_{3,0}, g_{4,1}\right)$. Unlike their two-dimensional projection, constraints (4.18), (4.20),

\footnotetext{
${ }^{9}$ Note that while the massless bosonic string amplitude, for example [17]

$$
\mathcal{A}_{\text {Bosonic }}\left(1^{+}, 2^{+}, 3^{+}, 4^{+}\right)=\frac{[12][34]}{\langle 12\rangle\langle 34\rangle} s t u\left(1-\frac{1}{s+1}-\frac{1}{t+1}-\frac{1}{u+1}\right) \frac{\Gamma(-s) \Gamma(-t)}{\Gamma(1-s-t)}
$$

also satisfies monodromy relations (3.8), the presence of Tachyon state will automatically result in violation of the Hankel matrix bound.
} 


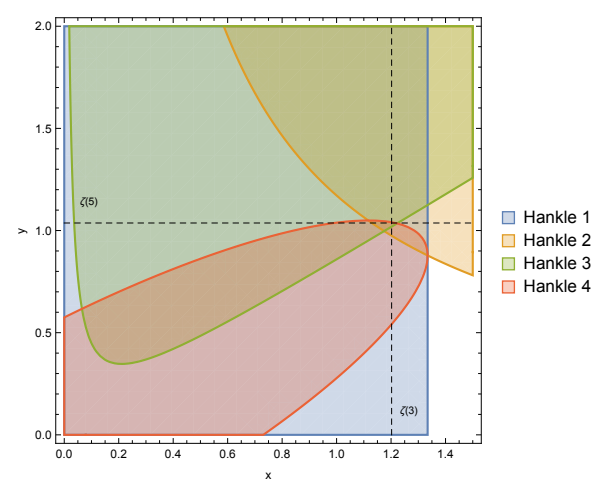

(a) Reduced result of all constraints up to $k=4$, intersection of $H_{1} \sim H_{4}$.

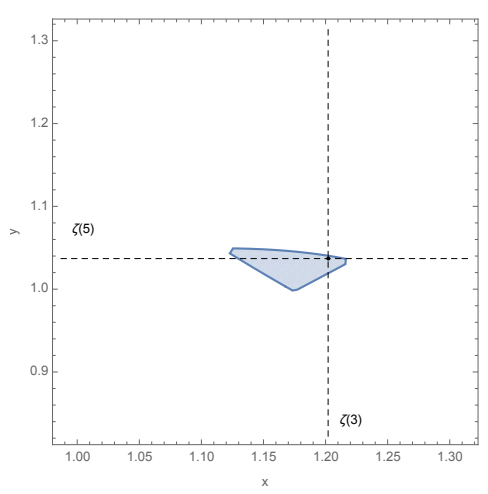

(b) Point corresponding to the superstring solution inside the carved region.

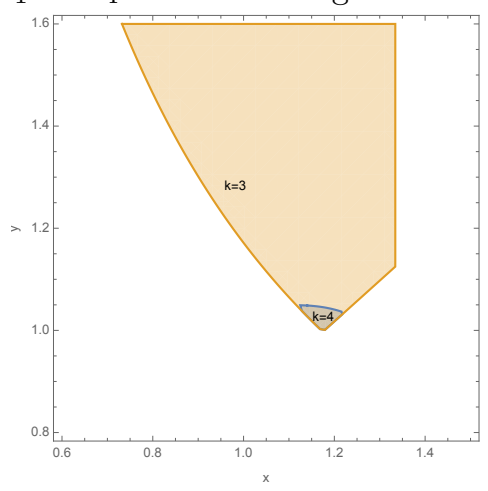

(c) Comparing carved region for $k=4$ with region for $k=3$.

Figure 8. Region on $(x, y)$-plane carved out by monodromy and EFThedron up to $k=4$. For simplicity we are only showing independent constraints after full reduction.

and (4.22) have nontrivial effect in carving this three-dimensional parameter space. An illustration for the three-dimensional intersection of all unitary constraints up to order $k=4,(4.8),(4.13),(4.15)$ as well as (4.16), (4.17), (4.20), (4.22), is presented in figure 9. It will serve as a starting data set for further investigations of compatibility with higher order unitary constraints.

\subsubsection{Peeking from beyond $k=4$}

Previously, we have seen that by considering $k=4$ constraints we further reduce the allowed region in $k \leq 3$. Here we will do the same at higher orders, to further restrict the region for $k=4$. Analytical reduction soon becomes impractical as often times the constraints are of higher algebraic order, and almost all new constraints needs to be projected. For example, at order $k=6$, we encounter the positive determinant condition for the Hankel matrix

$$
H_{9}=\left(\begin{array}{llll}
g_{0,0} & g_{1,0} & g_{2,0} & g_{3,0} \\
g_{1,0} & g_{2,0} & g_{3,0} & g_{4,0} \\
g_{2,0} & g_{3,0} & g_{4,0} & g_{5,0} \\
g_{3,0} & g_{4,0} & g_{5,0} & g_{6,0}
\end{array}\right),
$$




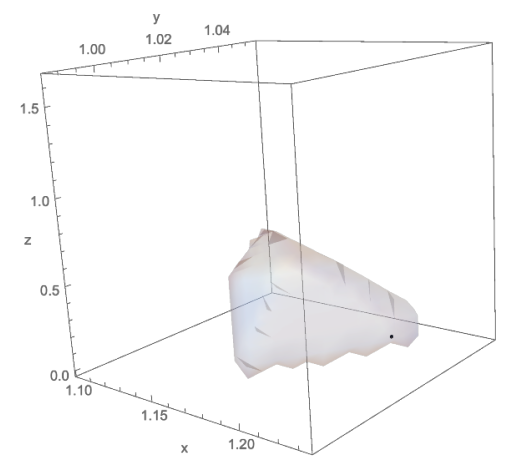

Figure 9. Region in $(x, y, z)$-space carved out by monodromy and EFT plane up to $k=4$. The marked point corresponded to the super string solution.

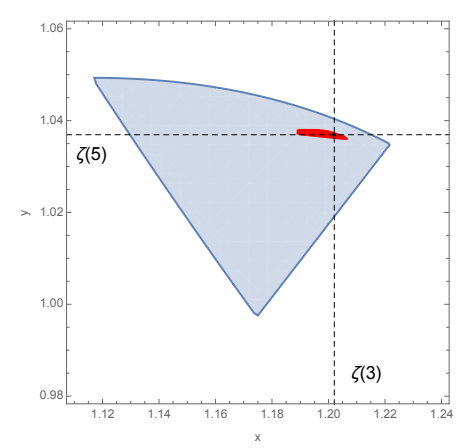

Figure 10. Projection of a subset of $k=6$ conditions. The blue region is the solution space carved out by monodromy and EFThedron up to $k=4$. It reduces to red region after projecting all $k=5$ constraints and one $k=6\left(\operatorname{det}\left(H_{9}\right)>0\right)$ constraint on $x, y$.

which is an order 3 inequality of free parameters $g_{1,0}=x, g_{3,0}=y$, and $g_{5,0}$. The projection onto the $(x, y)$-plane is obtained by solving for values of $x, y$ such that there exists a $g_{5,0}$ for which det $H_{9}>0$, i.e. points that can be uplifted into the $k=5$ geometry. Therefore, for order $k=6$ and beyond, for practical reasons, we will carry out the constraints in a numerical fashion and will only include the most relevant subset.

Our numerical survey reveals that even the inclusion of a subset of order $k=6$ constraints can give rise to much stronger conditions compared to the projection of $k=4$ constraints. We impose compatibility with constraints from the $k=5$ cyclic polytope, all Hankel and product Hankel matrices, as well as one $k=6$ matrix $H_{9}$ (4.23) on points in the blue region in figure $8 \mathrm{~b}$. We are able to rule out most of the region and achieve a much smaller allowed region, marked in red in figure 10. We plot within the $k=4$ lblue region to manifest the magnitude of this reduction.

We can perform the same scanning for points in figure 9 by imposing compatibility with $\operatorname{det}\left(H_{9}\right)>0$, as well as all positivity constraints for $k=5$. The result is again a more constrained region, which we show in red in figure 11a. We observe that, by including all constraints at $k=5$ and one at $k=6$, we have already fixed the value for $(x, y, z)$ to the 


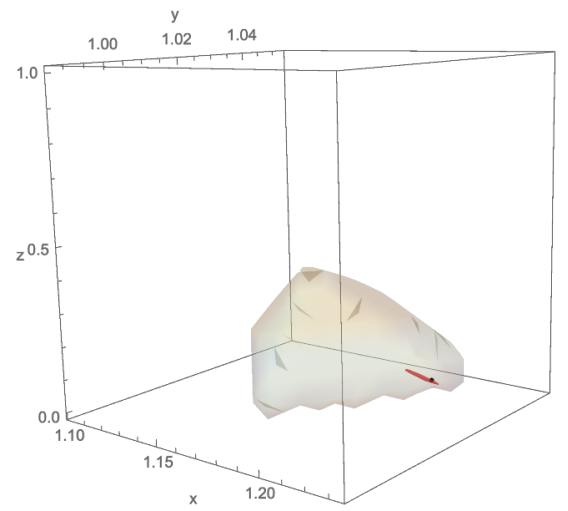

(a)

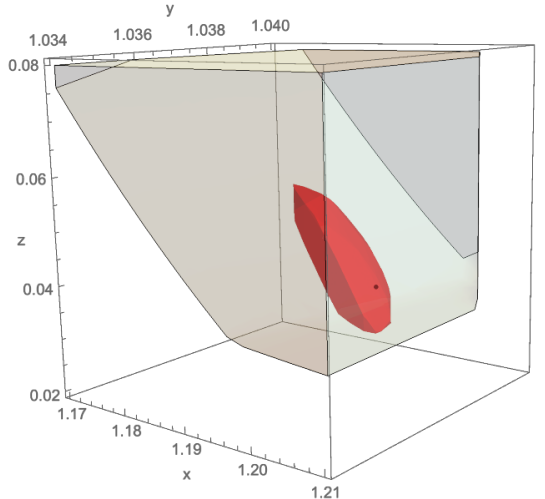

(b)

Figure 11. Carving the $k=4$ region by unitary constraints up to $k=4$. The gray region correspond to $k=4$ constraints and the red region is carved out by projecting $k=5$ and $\operatorname{det}\left(H_{9}\right)>0$ constraint. We can extract the ratio of red region width with respect to actual superstring results in each $(x, y, z)$ direction: $\frac{x^{\mathrm{max}}-x^{\mathrm{min}}}{x^{\mathrm{string}}} \approx 1.5 \%, \quad \frac{y^{\mathrm{max}}-y^{\mathrm{min}}}{y^{\text {string }}} \approx 0.2 \%, \quad \frac{z^{\mathrm{max}}-z^{\mathrm{min}}}{z^{\mathrm{string}}} \approx 52.8 \%$.

string value with the following precision:

$$
\begin{aligned}
& \frac{x^{\max }-x^{\min }}{x^{\text {string }}}=\frac{1.20667-1.18890}{\zeta(3)} \approx 1.5 \%, \\
& \frac{y^{\max }-y^{\min }}{y^{\text {string }}}=\frac{1.03808-1.03594}{\zeta(5)} \approx 0.2 \%, \\
& \frac{z^{\max }-z^{\min }}{z^{\text {string }}}=\frac{0.05699-0.03560}{\left.\left(\pi^{6}-630 \zeta(3)^{2}\right) / 1260\right)} \approx 52.8 \% .
\end{aligned}
$$

Extending to $k=8$, the reduction of constraints becomes difficult even with a numerical scan. However, we are able to use the FindInstance function in Mathematica to verify if a point in the $k=6$ allowed region of figure 10 or figure 11a is compatible with $k=7,8$ Hankel matrix constraints. We impose the positivity constraints for all $k=7$ Hankel matrices, and the following $k=8$ principal minor $H_{10}$

$$
\operatorname{det}\left(H_{10}\right)=\left(\begin{array}{lllll}
g_{0,0} & g_{1,0} & g_{2,0} & g_{3,0} & g_{4,0} \\
g_{1,0} & g_{2,0} & g_{3,0} & g_{4,0} & g_{5,0} \\
g_{2,0} & g_{3,0} & g_{4,0} & g_{5,0} & g_{6,0} \\
g_{3,0} & g_{4,0} & g_{5,0} & g_{6,0} & g_{7,0} \\
g_{4,0} & g_{5,0} & g_{6,0} & g_{7,0} & g_{8,0}
\end{array}\right)>0
$$

The allowed region on the $(x, y)$-plane and $(x, y, z)$-plane is now further reduced to the purple region in figure $12 \mathrm{a}$ and figure $12 \mathrm{~b}$ respectively.

As we find that the allowed regions of Laurent coefficients keep shrinking to the string value as we impose increasing order of unitarity constraints, we therefore conjecture that the full four-point open superstring amplitude $A(s, t)$ is uniquely determined by the intersection geometry of the monodromy plane with the EFThedron. 


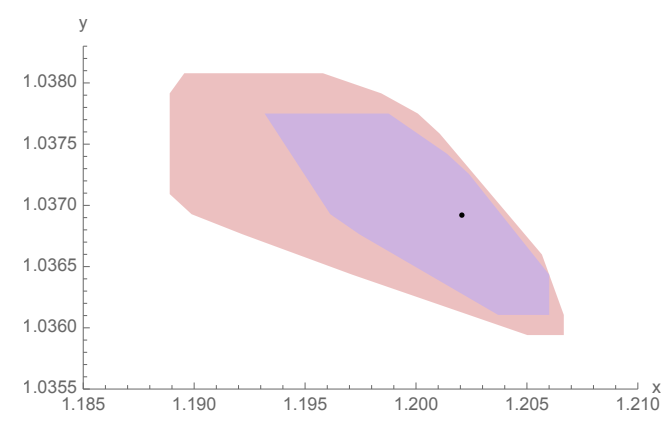

(a) Projecting $k=7$ and one $k=8$ constraint on $(x, y)$.

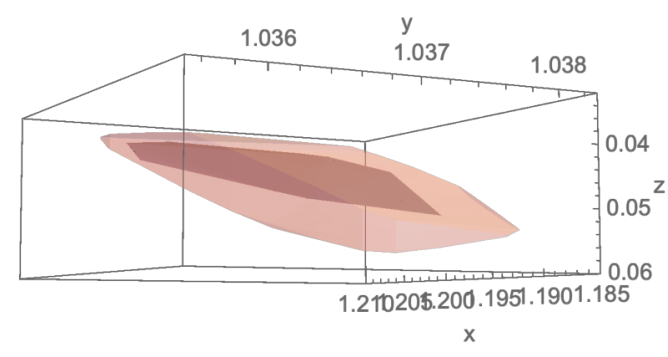

(b) Projecting $k=7$ and one $k=8$ constraint on $(x, y, z)$.

Figure 12. Region of parameter space curved out by projecting up to $k=7$ and $k=8$ constraints.

\subsection{Closed string EFT from KLT}

Given the monodromy relation of the open string amplitude, the closed string KLT relation [47] (see also [28, 29]) can be viewed as its corollary. Thus once we obtain the open string EFT, we can straightforwardly carve out the closed string EFT by projection through the KLT kernel. For example, combining (3.10) and (3.11) the projection through KLT yields the following:

$$
\begin{aligned}
M_{\text {closed string }}(s, t)= & A(s, t) \sin (\pi s) A(s,-s-t) \\
= & \frac{-\pi}{s t(s+t)}-2 \pi g_{1,0}-2 \pi g_{3,0}\left(s^{2}+s t+t^{2}\right) \\
& -\pi\left(\frac{\pi^{6}}{630}+g_{1,0}^{2}-2 g_{4,1}\right)(s+t) s t+2 \pi g_{5,0}\left(s^{2}+s t+t^{2}\right)^{2}+\ldots
\end{aligned}
$$

This can be compared to the EFT expansion

$$
M(s, t)=\frac{-\pi}{s t(s+t)}+\sum_{i, j=0}^{\infty} G_{i, j} s^{i-j} t^{i}
$$

where the coefficients $G_{i j}$ are linearly related to $g_{i, j}$ :

$$
\begin{aligned}
& G_{0,0}=2 \pi g_{1,0}, \quad G_{2,0}=G_{2,1}=G_{2,2}=2 \pi g_{3,0}, \\
& G_{3,1}=G_{3,2}=-\pi\left(\frac{\pi^{6}}{630}+g_{1,0}^{2}-2 g_{4,1}\right), \\
& G_{4,0}=G_{4,4}=2 \pi g_{5,0}, \quad G_{3,0}=G_{3,3}=0
\end{aligned}
$$

The shape of the allowed region of independent $G_{i, j}$ 's follows from the allowed region of monodromy free parameters $g_{1,0}, g_{3,0}, g_{4,1}$, etc. For example, the allowed region for the $G_{0,0}$, $G_{2,0}$ and $G_{3,1}$ is shown in figure 13, which is a straightforward coordinate transformation of the region in figure 11a which we used $(x, y, z)=\left(g_{1,0}, g_{3,0}, g_{4,1}\right)$. 


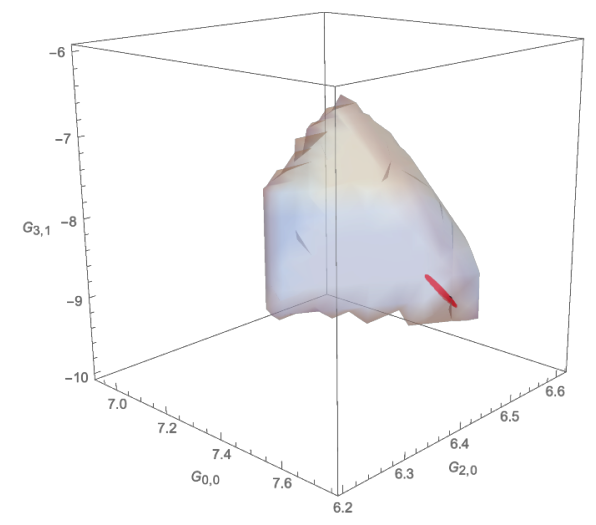

Figure 13. Region of parameter space $\left(G_{0,0}, G_{2,0}, G_{3,1}\right)$ for closed string in $\mathrm{d}=26$. The gray region carved out by monodromy and EFT up to $k=4$ constraints. The red region correspond to projected $k=5$ and $k=6$ condition.

The EFThedron constraint for non-ordered amplitude is much more intricate and not fully explored. However, in the forward limit, the Hankel matrix positivity is a direct carbon copy of the color ordered ones. Now the double copy in the forward limit takes the form:

$$
\begin{aligned}
& \lim _{t \rightarrow 0} A(s, t)(\pi t) A(-s, t) \\
& =\left(\frac{1}{\left(\alpha^{\prime}\right)^{2} s t}+\sum_{i, j=0}^{\infty} g_{i, 0} s^{i}\right)(\pi t)\left(\frac{1}{\left(\alpha^{\prime}\right)^{2}(-s) t}+\sum_{i, j=0}^{\infty} g_{i, 0}(-s)^{i}\right) .
\end{aligned}
$$

Only the contribution from odd powers of $s$ survives and the regular terms in $\mathcal{M}(s, t)$ read:

$$
-2 \pi\left(g_{1,0}+g_{3,0} s^{2}+g_{5,0} s^{4}+\ldots\right) .
$$

Thus we see that the Hankel positivity of the closed string amplitude is simply a subset of the open string ones, and the KLT kernel can be viewed as a positivity preserving projection. See also a similar discussion in [26].

\section{Conclusions and outlook}

In this paper, we study the interplay of consistency conditions for a space-time S-matrix, and a CFT four-point correlation function. This can be viewed as an initial step towards an on-shell approach to carving out the string landscape. By considering a worldsheet type integral representation for the four-point amplitude, we show that consistent factorization of the S-matrix forces the integrand to be given by linear combinations of $\mathrm{SL}(2, \mathrm{R})$ conformal blocks. Unitarity of the S-matrix for positive conformal weights carves out a subregion within the space of linear combinations, where we demonstrate that the Virasoro block appears at a kink in the boundary of allowed solutions. In the cases shown, this criteria is sufficient to analytically define the Virasoro combination. Thus Virasoro symmetry emerges from the consistency of the space-time S-matrix. 
Note that a characteristic property of $\chi_{q}$, defined as coefficients in the linear combination of global SL $(2, \mathrm{R})$ blocks for the Virasoro block, is the presence of poles associated with the null states. If instead we restrict ourselves to a polynomial ansatz, in general we can only cover a subspace of the allowed region, and it is only when we allow for a rational ansatz can we reach the boundary. Thus the presence of null state poles is crucial in putting us on the boundary. It will be interesting to understand more deeply from the S-matrix point of view the necessity for the presence of these null states.

In the opposite direction, we consider open string correlators with overall monodromy, which arrises for general flat-space amplitudes. We demonstrate that the resulting monodromy relations allow for three isolated solutions, each enforcing algebraic identities amongst the low energy couplings. Thus the low energy description of string theory amplitudes corresponds to the intersection of the "monodromy plane" with the EFThedron. For the monodromy plane that arises for usual flat space amplitudes, we show that the intersection space is an isolated island, whose area rapidly converges as higher derivative order constraints are taken into account. This leads us to conjecture that the four-point open superstring amplitude is completely fixed by the geometry of the intersection between the monodromy plane and the EFThedron. This also implies that deviation from the superstring result must entail modifications to the monodromy relations employed. We present the result for the same investigation on bicolor monodromy.

Since the intersection geometry is infinite dimensional in nature, it will be desirable to have a continuous limit description. Note that this is reminiscent of the CFT bootstrap, where the initial derivative truncation gave way to more efficient analytic functionals [48]. The initial step would be then to have a continuous definition for the Hankel matrix bounds. A recent proposal of positive functionals is a very promising direction [49].

The constrained space of open string EFT couplings naturally leads to a constrained space for closed strings couplings through KLT relations. We find that so long as the open string couplings reside in the EFThedron, the closed string image automatically satisfies all Hankel-type bounds. However, it is well known that the EFThedron for general permutation invariant theories is much more intricate than the color ordered ones [26]. It will be interesting in the future to see if the KLT kernel always projects the intersection geometry of the open string inside the general EFThedron. It is clearly desirable to understand what statements can be made for the monodromy of general string compactifications, and study how to modify our approach to cases where instead of universal monodromy, the open string amplitude is given as sum of blocks with distinct yet understood monodromy phases. Finally, it was recently shown that the string EFT expansion can be expressed in terms of just a few modified color-kinematic building blocks [50]. It would be interesting to understand how monodromy relates the color-kinematic solutions at different mass dimension, and if monodromy-compatible solutions themselves are amenable to a direct bootstrap procedure. 


\section{Acknowledgments}

We would like to thank Nima Arkani-Hamed, Shu-Heng Shao and Pierre Vanhove for discussions at the early stages of this work, Chi-Ming Chang for enlightening discussions, Yang Zhang for helping improve efficiency of the algorithm, and John Joseph Carrasco and Congkao Wen for comments on the draft. Y.-h. Wang is supported by MoST Grant No. 108-2811-M-002-535. Y.-t. Huang and J.-y. Liu are supported by MoST Grant No. 1062628-M-002-012-MY3. Y.-t. Huang is also supported by Golden Jade fellowship. L. Rodina is supported by the European Research Council under ERC-STG-639729, Strategic Predictions for Quantum Field Theories.

\section{A Expanding coefficient}

The scalar coefficient in $n=2$

$$
\begin{aligned}
\mathcal{C}_{0}^{(2)}= & \frac{d(p+1)^{2}}{8(d-1)}+\frac{d\left(i_{1}-i_{2}\right)^{2}\left(i_{3}-i_{4}\right)^{2}}{8(d-1)(p+1)^{2}}-\frac{(p+1)\left(d-5+d\left(i_{1}-i_{2}-i_{3}+i_{4}\right)+2\left(i_{2}+i_{3}\right)\right)}{4(d-1)}, \\
& +\frac{d}{8(d-1)}\left(i_{1}^{2}-2 i_{2} i_{1}-4 i_{3} i_{1}+4 i_{4} i_{1}+2 i_{1}+i_{2}^{2}+i_{3}^{2}+i_{4}^{2}-2 i_{2}+4 i_{2} i_{3}-2 i_{3}-4 i_{2} i_{4}-2 i_{3} i_{4}+2 i_{4}\right) \\
& +\frac{1}{4(d-1)}\left(4 i_{3} i_{1}-5 i_{1}-3 i_{2}-3 i_{3}+4 i_{2} i_{4}-5 i_{4}+8\right) \\
& +\frac{1}{4(d-1)(p+1)}\left(i_{1}^{2}\left((d-2) i_{3}-d i_{4}+2\right)-i_{1}\left(2 i_{2}\left((d-2) i_{3}-d i_{4}+2\right)+\left(i_{3}-i_{4}\right)\left(d i_{3}-d i_{4}-d+1\right)\right)\right. \\
& \left.+i_{2}\left(i_{3}-i_{4}\right)\left((d-2) i_{3}-(d-2) i_{4}-d+1\right)+i_{2}^{2}\left((d-2) i_{3}-d i_{4}+2\right)+2\left(i_{3}-i_{4}\right)^{2}\right) \\
& +\frac{\left(i_{1}-i_{2}-p-1\right)\left(i_{3}-i_{4}+p+1\right)}{2(p+1)} v_{1}+v_{2} .
\end{aligned}
$$

\section{B Virasoro block}

In this appendix, we list the ansatz we used in section 2.2 and show the detail for fixing ansatz parameters in $n=2$ and $n=3$.

$\boldsymbol{n}=\mathbf{2} . \quad$ Ansatz of $\chi_{2}$ :

$$
\chi_{2}=\frac{\left(a_{1} i_{1}+a_{2} i_{2}+a_{3} i_{1}^{2}+a_{4} i_{2}^{2}+a_{5} i_{1} i_{2}+a_{6} p+a_{7} i_{1} p+a_{8} i_{2} p+a_{9} p^{2}\right)\left(i_{1} \rightarrow i_{4}, i_{2} \rightarrow i_{3}\right)}{\left(b_{0}+b_{1} d+b_{2} p+b_{3} d p+b_{4} p^{2}+b_{5} d p^{2}+b_{6} p^{3}\right)} .
$$

Note that we have allowed the dependence on $i_{1}, i_{2}, i_{3}, i_{4}$ and $p$ up to at least degree 2 in the numerator, as there are no solutions to $(2.27),(2.28)$ with lower degrees. We find that simply requiring the scalar coefficient to satisfy the equality in (2.28) for all external dimensions, while respecting (2.27), the $\chi_{2}$ ansatz in (B.1) is reduced to

$$
\chi_{2}=\frac{\left(3 i_{1}^{2}+3 i_{2}^{2}-i_{2}(1+6 a p)-i_{1}(1+6 a p)-6 i_{1} i_{2}+p(-5-3 p+6 x(3+p))\right)\left(i_{1} \rightarrow i_{4}, i_{2} \rightarrow i_{3}\right)}{\left.\left(2 d(1+6 a p)^{2}-4(1+p)\left(13-6(-7+4 a) p+36(-1+a)^{2} p^{2}\right)\right)\right)},
$$

where the variable $a$ is the ratio $a=a_{7} / a_{5}$. This is constrained to $-1.25 \leq a \leq 1 / 3$ as we now see. Note that the coefficient $\mathcal{C}_{0}^{(2)}$, with $\chi_{2}$ given in (B.2), becomes:

$$
\mathcal{C}_{0}^{(2)}=\frac{\mathcal{N}\left(i_{1}, i_{2}, p, a\right) \mathcal{N}\left(i_{4}, i_{3}, p, a\right)}{8(-1+d)(1+p)^{2}(1+2 p)\left(-d(1+6 p a)^{2}+2(1+p)\left(13-6(-7+4 a) p+36(-1+a)^{2} p^{2}\right)\right)} .
$$


If one requires the coefficient to be positive when $i_{1}=i_{4}, i_{2}=i_{3}$, then the denominator must be positive

$$
-d(1+6 p a)^{2}+2(1+p)\left(13-6(-7+4 a) p+36(a-1)^{2} p^{2}\right) \geq 0 .
$$

It is easy to see positivity for positive $p$ in dimensions below $d=26$ will bound $-1.25 \leq$ $a \leq 1 / 3$. We can consider the positivity constraint at higher order $n$. For example, the positivity of $\mathcal{C}_{1}^{(3)}$ will further restrict the region to $0.32 \leq a \leq 1 / 3$. As the lower bound asymptotes to match with the upper bound, fixed at $1 / 3$, being at the boundary of $(2.27),(2.28)$ uniquely determines $\chi_{2}$ to be:

$$
\chi_{2}=\frac{\left(-3\left(i_{1}-i_{2}\right)^{2}+\left(i_{1}+i_{2}\right)+2 p\left(i_{1}+i_{2}\right)+(p-1) p\right)\left(-3\left(i_{4}-i_{3}\right)^{2}+\left(i_{4}+i_{3}\right)+2 p\left(i_{4}+i_{3}\right)+(p-1) p\right)}{2(1+2 p)\left(26-d+42 p-2 d p+16 p^{2}\right)} .
$$

$\boldsymbol{n}=3 . \quad$ Ansatz of $\chi_{3}$ :

$$
\begin{aligned}
\chi_{3}=- & \frac{T\left(i_{1}, i_{2}\right) T\left(i_{4}, i_{3}\right)}{b_{0}+b_{1} p+b_{2} p^{2}+b_{3} p^{3}+b_{4} p^{4}+b_{5} p^{5}+b_{6} d+b_{7} p d+b_{8} p^{2} d+b_{9} p^{3} d+b_{10} p^{4} d} \\
T(i, j)= & \left(a_{1} i^{2}+a_{2} j^{2}+a_{3} i j+a_{4} i^{3}+a_{5} i^{2} j+a_{6} i j^{2}+a_{7} j^{3}+a_{8} i p\right. \\
& \left.+a_{9} j p+a_{10} i^{2} p+a_{11} j^{2} p+a_{12} i j p+a_{13} i p^{2}+a_{14} j p^{2}\right)
\end{aligned}
$$

Setting $\chi_{1}=0$ and $\chi_{2}$ to (2.33), once again by imposing (2.27) and equality in (2.28) for $\mathcal{C}_{0}^{(3)}$, the ansatz for $\chi_{3}$ can be completely fixed up to the ratio $a=a_{11} / a_{2}$, which is confined to the region $-6.58 \leq a \leq 1$. This range is considerably reduced by considering the positivity in (2.27) for $\mathcal{C}_{1}^{(4)}$, restricting to $0.95 \leq a \leq 1$. The positivity of $C_{n-3}^{(n)}$ in (2.27) at higher $n$ further pushes the lower bound to 1, thus fixing all the ansatz to:

$$
\chi_{3}=-\frac{\left(i_{1}-i_{2}\right)\left(i_{3}-i_{4}\right)\left(-i_{1}+i_{1}^{2}-i_{2}+i_{2}^{2}-2 i_{1} i_{2}+p-i_{1} p-i_{2} p\right)\left(-i_{3}+i_{3}^{2}-i_{4}+i_{4}^{2}-2 i_{3} i_{4}+p-i_{3} p-i_{4} p\right)}{2 p(p+1)(p+2)\left(28-d+19 p-d p+3 p^{2}\right)} .
$$

\section{Bicolor ordered amplitudes}

In this section we carry out the analysis for a bi-adjoint effective theory, and show that monodromy and EFT-hedron constraints imply it must match Z-theory [15, 16].

\section{C.1 BCJ and monodromy plane}

Such amplitudes have a doubly-ordered structure of the form $\mathcal{A}(\mathcal{P} \mid \mathcal{Q})$, where $\mathcal{P}, \mathcal{Q}$ are two permutations of the color indices $\{1,2,3,4\}$.

We will impose that the amplitude satisfies BCJ relations with respect to permutations of the set $\mathcal{P}$,

$$
u \mathcal{A}(1324 \mid 1234)=s \mathcal{A}(1234 \mid 1234)
$$


and the monodromy relation with respect to the set $\mathcal{Q}$,

$$
\mathcal{A}(1234 \mid 2134)+e^{i \pi s} \mathcal{A}(1234 \mid 1234)+e^{-i \pi u} \mathcal{A}(1234 \mid 1324)=0 .
$$

This is required if the amplitude originates from a worldsheet expression of the following form

$$
\begin{aligned}
\mathcal{A}(\mathcal{P} \mid \mathcal{Q})= & -S\left(\left\{k_{i}, \epsilon_{i}\right\}\right) \int_{0}^{1} d z_{\mathcal{P}(2)} f\left(z_{i}\right) z_{\mathcal{P}(2)}^{-2 k_{\mathcal{P}(1)} \cdot k_{\mathcal{P}(2)}}\left(1-z_{\mathcal{P}(2)}\right)^{-2 k_{\mathcal{P}(2)} \cdot k_{\mathcal{P}(3)}} \\
& \times \frac{\left(z_{\mathcal{P}(1)}-z_{\mathcal{P}(3)}\right)\left(z_{\mathcal{P}(1)}-z_{\mathcal{P}(4)}\right)\left(z_{\mathcal{P}(3)}-z_{\mathcal{P}(4)}\right)}{\prod_{i=1}^{4}\left(z_{\mathcal{Q}(i)}-z_{\mathcal{Q}(i+1)}\right)}
\end{aligned}
$$

where $f\left(z_{i}\right)$ is a total symmetric function of all $z_{i}$ 's, and has trivial monodromy.

For such bicolor amplitudes satisfying BCJ and monodromy relations there is only one independent four-point amplitude, all other amplitudes with different orders of the two sets of indices can be derived by repeated use of the BCJ relation and/or the monodromy relation. Therefore, as in the single color case, we only need to consider the parameter space of one amplitude, which we can choose to be the symmetric one $\mathcal{A}$ (1234|1234). This has the same form as (3.7)

$$
\mathcal{A}(1234 \mid 1234)=S\left(\left\{k_{i}, \epsilon_{i}\right\}\right) A(s, t)
$$

First of all, under permutations of the external legs, we have

$$
\begin{aligned}
& S\left(\left\{k_{i}, \epsilon_{i}\right\}\right) A(s, t)=\mathcal{A}(1234 \mid 1234), \\
& S\left(\left\{k_{i}, \epsilon_{i}\right\}\right) A(u, t)=\mathcal{A}(1324 \mid 1324), \\
& S\left(\left\{k_{i}, \epsilon_{i}\right\}\right) A(s, u)=\mathcal{A}(2134 \mid 2134),
\end{aligned}
$$

by unifying the first color order by applying BCJ relation:

$$
\begin{aligned}
\frac{u}{t} \mathcal{A}(2134 \mid 2134) & =\mathcal{A}(1234 \mid 2134), \\
\frac{u}{s} \mathcal{A}(1324 \mid 1324) & =\mathcal{A}(1234 \mid 1324),
\end{aligned}
$$

and comparing with (C.2), we arrive at

$$
\frac{u}{s} A(s, u)+e^{i \pi s} A(s, t)+e^{-i \pi u} \frac{t}{s} A(u, t)=0 .
$$

The Laurent expansion of $A(s, t)$ is

$$
A(s, t)=-\left(\frac{1}{s}+\frac{1}{t}\right)+\sum_{k \geq q \geq 0} g_{k, q} s^{k-q} t^{q},
$$


and by solving the monodromy relation we find the coefficients:

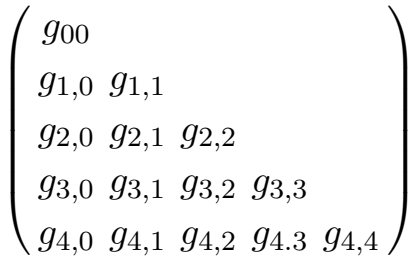

$$
\begin{aligned}
& =\left(\begin{array}{cccc}
0 & & & \\
\zeta(2) & \zeta(2) & g_{2,0} & \\
g_{2,0} & 2 g_{2,0} & \frac{5}{4} \zeta(4) & \zeta(4) \\
\zeta(4) & \frac{5}{4} \zeta(4) & g_{2,0}+4 g_{4,0}-\zeta(2) g_{2,0}+3 g_{4,0} & g_{4,0} .
\end{array}\right)
\end{aligned}
$$

Note that in (C.10) we are in fact solving for the Laurent expansion of $(s+t) A(s, t)$ for $A(s, t)$ in (3.8), so the parameters in (C.12) and (3.11) are in one-to-one correspondence as expected. Naturally the correspondence between monomials of odd zeta values and free parameters emerges in the bicolor case as well.

\section{C.2 Combined constraints for bicolor ordered amplitude}

For the bicolor amplitude (C.11), the Laurent coefficients $g_{i, j}$ are constrained by the combination of unitarity and monodromy in a similar fashion. In this subsection we will show the result for the combined constraints on $\left(g_{2,0}, g_{4,0}\right)=(x, y)$ up to $k=5$. As shown in (C.11), the monodromy constraints read:

$$
\begin{array}{lll}
k=0: & g_{0,0}=0 \\
k=1: & g_{1,0}=g_{1,1}=\zeta(2), & \\
k=2: & g_{2,1}=2 g_{2,2}=2 g_{2,0}, & \\
k=3: & g_{3,0}=g_{3,3}=\zeta(4), & g_{3,1}=g_{3,2}=\frac{5}{4} \zeta(4), \\
k=4: & g_{4,1}=g_{4,3}=-\zeta(2) g_{2,0}+3 g_{4.0}, & g_{4,2}=-2 \zeta(2) g_{2,0}+4 g_{4,0} .
\end{array}
$$

Thus the monodromy plane can be identified with the plane spanned by $\left(g_{2,0}, g_{4,0}\right)=$ $(x, y)$. The cyclic polytope (3.23) expanded on the monodromy plane will give us some non-relevant conditions, which we do not list here. One nontrivial constraint is that the components of $\vec{g}_{4}$ should be positive. The positivity of the third component in $\vec{g}_{4}^{(3)}=$ $g_{4,2} / g_{4,0}$ can be expanded on the monodromy plane and implies:

$$
\frac{g_{4,2}}{g_{4,0}}>0 \longrightarrow \frac{y}{x}>\frac{\pi^{2}}{12}
$$




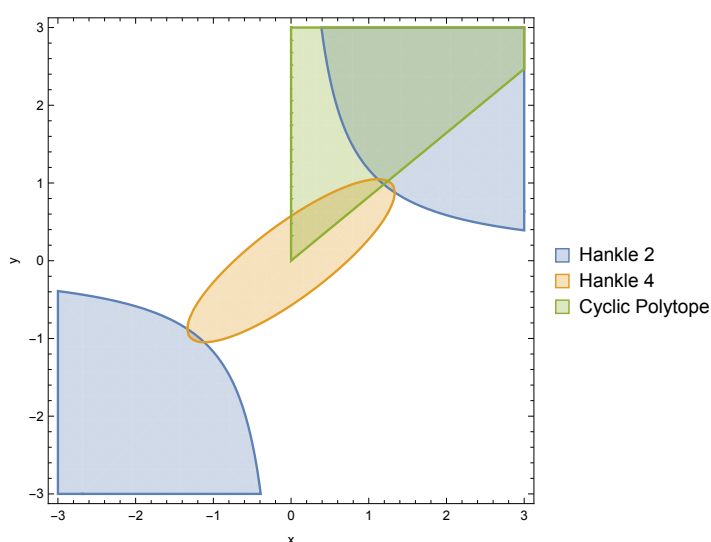

(a) The constraint of cyclic Polytope and Hankel matrix. We list the strongest condition up to $k=5$.

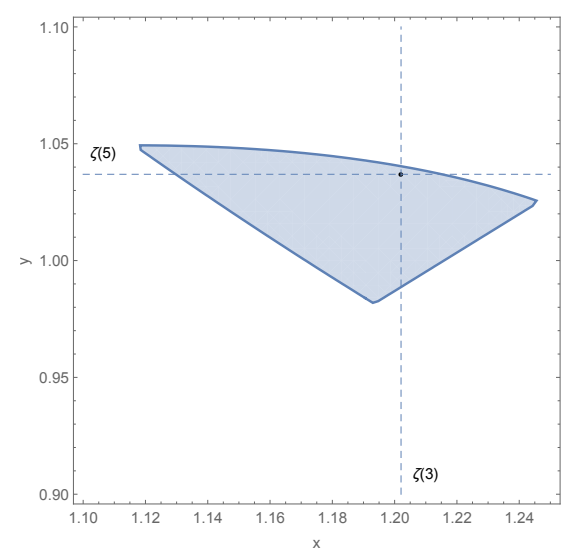

(b) The region of intersection and position of $\mathrm{Z}$ amplitude solution.

Figure 14. Region of parameter space for bi-color amplitude carved out by monodromy and EFT plane.

As we know $g_{5,0}=\zeta(6)$ from monodromy, we can include positive determinant constraints for Hankel matrices (3.24) containing $g_{5,0}$

$$
\begin{array}{lll}
H_{1}=\left(\begin{array}{ll}
g_{1,0} & g_{2,0} \\
g_{2,0} & g_{3,0}
\end{array}\right), & H_{2}=\left(\begin{array}{ll}
g_{2,0} & g_{3,0} \\
g_{3,0} & g_{4,0}
\end{array}\right), & H_{3}=\left(\begin{array}{ll}
g_{2,1} & g_{3,1} \\
g_{3,1} & g_{4,1}
\end{array}\right) \\
H_{4}=\left(\begin{array}{lll}
g_{1,0} & g_{2,0} & g_{3,0} \\
g_{2,0} & g_{3,0} & g_{4,0} \\
g_{3,0} & g_{4,0} & g_{5,0}
\end{array}\right), & H_{5}=\left(\begin{array}{ll}
g_{3,0} & g_{4,0} \\
g_{4,0} & g_{5,0}
\end{array}\right), & H_{6}=\left(\begin{array}{ll}
g_{1,0} & g_{3,0} \\
g_{3,0} & g_{5,0}
\end{array}\right) .
\end{array}
$$

The product Hankel matrices (3.25) are also not relevant in this case. We summarize all the nontrivial constraints in (C.13), (C.14) and the matrices $\operatorname{det}\left(H_{i}\right)>0$ in (C.16) in figure 14a. As in the single color case, the parameters are confined to a small region, shown in figure $14 \mathrm{~b}$.

\section{Checking a corollary of the main conjecture}

Verifying the conjecture becomes computationally difficult at higher orders in $k$, but we can test a simpler yet non-trivial corollary, with significant precision. It is a straightforward consequence of the conjecture that for a EFT amplitude with some of its Laurent coefficients set equal the string value, the remaining unknown parameters can be fixed by monodromy and unitarity to the string value as well.

By setting $\left(g_{1,0}, g_{3,0}, g_{4,1}\right)$ to their string values $\left(\zeta(3), \zeta(5), 3 / 4 \zeta(6)-1 / 2 \zeta(3)^{2}\right)$, we can search for solutions of the higher order parameters to the $k=7$ EFThedron constraints via FindInstance. We immediately find:

$$
\left(g_{5,0}, g_{6,1}, g_{7,0}, g_{7,2}\right)=(1.00834,0.00862,1.00202,0.00035),
$$


matching the string values up to four digits:

$$
\begin{aligned}
& \left(\zeta(7), \frac{\pi^{8}}{7560}-\zeta(3) \zeta(5), \zeta(9), \frac{180 \zeta(3)^{3}-2 \pi^{6} \zeta(3)-27 \pi^{4} \zeta(5)-540 \pi^{2} \zeta(7)+10080 \zeta(9)}{1080}\right) \\
& =(1.00835,0.00865,1.00201,0.00032) .
\end{aligned}
$$

The most manageable case of this corollary is when all but one parameter is fixed to the string value. For example, at $k=5$ monodromy leaves four independent variables. We can deform $g_{1,0}$, and keep the remaining parameters at the superstring value:

$$
\begin{aligned}
g_{1,0} & =\zeta(3)+x \\
g_{3,0} & =\zeta(5) \\
g_{4,1} & =\frac{3}{4} \zeta(6)-\frac{1}{2} \zeta(3)^{2} \\
g_{5,0} & =\zeta(7),
\end{aligned}
$$

including all higher order $k$ couplings. Our conjecture implies that as we increase the order of the constraints, $x$ should be bounded closer and closer to zero. We restrict to just using the following choice of Hankel matrices for simplicity:

$$
H_{n}=\left(\begin{array}{cc}
g_{n, i} & g_{n+1, i} \\
g_{n+1, i} & g_{n+2, i}
\end{array}\right)
$$

for all $i \leq n$, and we impose all entries to be positive, along with $\operatorname{det}\left(H_{n}\right)>0$. Even with this drastically reduced set of constraints we find that the value of $x$ quickly becomes highly constrained:

$$
\begin{aligned}
H_{5}: & -10^{-4}<x<10^{-3}, \\
H_{7}: & -10^{-7}<x<10^{-3} \\
H_{9}: & -10^{-9}<x<10^{-6}, \\
H_{11}: & -10^{-11}<x<10^{-8} .
\end{aligned}
$$

Another easy test can be done for the case when we deform all $\zeta(3) \rightarrow \zeta(3)+x$, including any $\zeta(3)^{k} \rightarrow(\zeta(3)+x)^{k}$, for all $k$. Unlike the first test, this will put bounds on $\zeta(3)$ purely in terms of other zeta values. In this case we also get an increase in precision with constraint order:

$$
\begin{aligned}
H_{5}: & -10^{-4}<x<10^{0}, \\
H_{7}: & -10^{-5}<x<10^{-1}, \\
H_{9}: & -10^{-6}<x<10^{-2}, \\
H_{11}: & -10^{-7}<x<10^{-3},
\end{aligned}
$$

suggesting we can indeed fix $\zeta(3)$ to arbitrary degree.

Open Access. This article is distributed under the terms of the Creative Commons Attribution License (CC-BY 4.0), which permits any use, distribution and reproduction in any medium, provided the original author(s) and source are credited. 


\section{References}

[1] R. Rattazzi, V.S. Rychkov, E. Tonni and A. Vichi, Bounding scalar operator dimensions in $4 D$ CFT, JHEP 12 (2008) 031 [arXiv:0807.0004] [INSPIRE].

[2] D. Simmons-Duffin, The Conformal Bootstrap, in Theoretical Advanced Study Institute in Elementary Particle Physics: New Frontiers in Fields and Strings, (2016) [DOI] [arXiv: 1602.07982] [INSPIRE].

[3] D. Poland and D. Simmons-Duffin, The conformal bootstrap, Nature Phys. 12 (2016) 535 [INSPIRE].

[4] D. Poland, S. Rychkov and A. Vichi, The Conformal Bootstrap: Theory, Numerical Techniques, and Applications, Rev. Mod. Phys. 91 (2019) 015002 [arXiv:1805.04405] [INSPIRE].

[5] M.F. Paulos, J. Penedones, J. Toledo, B.C. van Rees and P. Vieira, The S-matrix bootstrap. Part I: QFT in AdS, JHEP 11 (2017) 133 [arXiv:1607.06109] [INSPIRE].

[6] M.F. Paulos, J. Penedones, J. Toledo, B.C. van Rees and P. Vieira, The S-matrix bootstrap II: two dimensional amplitudes, JHEP 11 (2017) 143 [arXiv:1607.06110] [INSPIRE].

[7] M.F. Paulos, J. Penedones, J. Toledo, B.C. van Rees and P. Vieira, The S-matrix bootstrap. Part III: higher dimensional amplitudes, JHEP 12 (2019) 040 [arXiv:1708.06765] [INSPIRE].

[8] A. Homrich, J. Penedones, J. Toledo, B.C. van Rees and P. Vieira, The S-matrix Bootstrap IV: Multiple Amplitudes, JHEP 11 (2019) 076 [arXiv: 1905.06905] [INSPIRE].

[9] S.D. Chowdhury, A. Gadde, T. Gopalka, I. Halder, L. Janagal and S. Minwalla, Classifying and constraining local four photon and four graviton S-matrices, JHEP 02 (2020) 114 [arXiv:1910.14392] [INSPIRE].

[10] A. Bose, P. Haldar, A. Sinha, P. Sinha and S.S. Tiwari, Relative entropy in scattering and the S-matrix bootstrap, SciPost Phys. 9 (2020) 081 [arXiv:2006.12213] [INSPIRE].

[11] F. Cachazo, S. He and E.Y. Yuan, Scattering of Massless Particles in Arbitrary Dimensions, Phys. Rev. Lett. 113 (2014) 171601 [arXiv:1307.2199] [INSPIRE].

[12] F. Cachazo, S. He and E.Y. Yuan, Scattering of Massless Particles: Scalars, Gluons and Gravitons, JHEP 07 (2014) 033 [arXiv: 1309.0885] [INSPIRE].

[13] F. Cachazo, S. He and E.Y. Yuan, Scattering Equations and Matrices: From Einstein To Yang-Mills, DBI and NLSM, JHEP 07 (2015) 149 [arXiv:1412.3479] [INSPIRE].

[14] C.R. Mafra, O. Schlotterer and S. Stieberger, Complete N-Point Superstring Disk Amplitude I. Pure Spinor Computation, Nucl. Phys. B 873 (2013) 419 [arXiv:1106.2645] [INSPIRE].

[15] J.J.M. Carrasco, C.R. Mafra and O. Schlotterer, Abelian Z-theory: NLSM amplitudes and $\alpha$ '-corrections from the open string, JHEP 06 (2017) 093 [arXiv: 1608.02569] [INSPIRE].

[16] C.R. Mafra and O. Schlotterer, Non-abelian Z-theory: Berends-Giele recursion for the $\alpha^{\prime}$-expansion of disk integrals, JHEP 01 (2017) 031 [arXiv:1609.07078] [INSPIRE].

[17] Y.-t. Huang, O. Schlotterer and C. Wen, Universality in string interactions, JHEP 09 (2016) 155 [arXiv: 1602.01674] [INSPIRE].

[18] T. Azevedo, M. Chiodaroli, H. Johansson and O. Schlotterer, Heterotic and bosonic string amplitudes via field theory, JHEP 10 (2018) 012 [arXiv: 1803.05452] [INSPIRE]. 
[19] N. Arkani-Hamed, S. He and T. Lam, Stringy canonical forms, JHEP 02 (2021) 069 [arXiv: 1912.08707] [INSPIRE].

[20] A. Adams, N. Arkani-Hamed, S. Dubovsky, A. Nicolis and R. Rattazzi, Causality, analyticity and an IR obstruction to UV completion, JHEP 10 (2006) 014 [hep-th/0602178] [INSPIRE].

[21] B. Bellazzini, C. Cheung and G.N. Remmen, Quantum Gravity Constraints from Unitarity and Analyticity, Phys. Rev. D 93 (2016) 064076 [arXiv:1509.00851] [InSPIRE].

[22] C. de Rham, S. Melville, A.J. Tolley and S.-Y. Zhou, Positivity bounds for scalar field theories, Phys. Rev. D 96 (2017) 081702 [arXiv:1702.06134] [InSPIRE].

[23] C. de Rham, S. Melville and A.J. Tolley, Improved Positivity Bounds and Massive Gravity, JHEP 04 (2018) 083 [arXiv: 1710.09611] [INSPIRE].

[24] W.-M. Chen, Y.-T. Huang, T. Noumi and C. Wen, Unitarity bounds on charged/neutral state mass ratios, Phys. Rev. D 100 (2019) 025016 [arXiv:1901.11480] [INSPIRE].

[25] N. Arkani-Hamed, T.-C. Huang and Y.-T. Huang, The EFT-Hedron, arXiv:2012.15849 [INSPIRE].

[26] M.B. Green and C. Wen, Superstring amplitudes, unitarily, and Hankel determinants of multiple zeta values, JHEP 11 (2019) 079 [arXiv:1908.08426] [INSPIRE].

[27] E. Plahte, Symmetry properties of dual tree-graph n-point amplitudes, Nuovo Cim. A 66 (1970) 713 [INSPIRE].

[28] N.E.J. Bjerrum-Bohr, P.H. Damgaard and P. Vanhove, Minimal Basis for Gauge Theory Amplitudes, Phys. Rev. Lett. 103 (2009) 161602 [arXiv:0907.1425] [INSPIRE].

[29] S. Stieberger, Open 83 Closed vs. Pure Open String Disk Amplitudes, arXiv:0907.2211 [INSPIRE].

[30] R.H. Boels and T. Hansen, String theory in target space, JHEP 06 (2014) 054 [arXiv: 1402.6356] [INSPIRE].

[31] D.D. Coon, Uniqueness of the veneziano representation, Phys. Lett. B 29 (1969) 669 [INSPIRE].

[32] S. Matsuda, Uniqueness of the veneziano representation, Phys. Rev. 185 (1969) 1811 [INSPIRE].

[33] N.N. Khuri, Derivation of a veneziano series from the Regge representation, Phys. Rev. 185 (1969) 1876 [INSPIRE].

[34] E. Weimar, Alternatives to the Veneziano Amplitude, DESY-74-3 [INSPIRE].

[35] P.G.O. Freund, finite energy sum rules and bootstraps, Phys. Rev. Lett. 20 (1968) 235 [INSPIRE].

[36] M. Froissart, Asymptotic behavior and subtractions in the Mandelstam representation, Phys. Rev. 123 (1961) 1053 [INSPIRE].

[37] D.J. Gross and P.F. Mende, The High-Energy Behavior of String Scattering Amplitudes, Phys. Lett. B 197 (1987) 129 [INSPIRE].

[38] D.J. Gross and J.L. Manes, The High-energy Behavior of Open String Scattering, Nucl. Phys. B 326 (1989) 73 [INSPIRE]. 
[39] S. Caron-Huot, Z. Komargodski, A. Sever and A. Zhiboedov, Strings from Massive Higher Spins: The Asymptotic Uniqueness of the Veneziano Amplitude, JHEP 10 (2017) 026 [arXiv: 1607.04253] [INSPIRE].

[40] E. Perlmutter, Virasoro conformal blocks in closed form, JHEP 08 (2015) 088 [arXiv: 1502.07742] [INSPIRE].

[41] M. Bianchi, D. Consoli and P. Di Vecchia, On the N-pion extension of the Lovelace-Shapiro model, JHEP 03 (2021) 119 [arXiv : 2002. 05419] [INSPIRE].

[42] R. Kleiss and H. Kuijf, Multi-Gluon Cross-sections and Five Jet Production at Hadron Colliders, Nucl. Phys. B 312 (1989) 616 [INSPIRE].

[43] Z. Bern, J.J.M. Carrasco and H. Johansson, New Relations for Gauge-Theory Amplitudes, Phys. Rev. D 78 (2008) 085011 [arXiv:0805.3993] [InSPIRE].

[44] T. Terasoma, Selberg Integrals and Multiple Zeta Values, Compos. Math. 133 (2002) 1 [math/9908045].

[45] S. Stieberger, Constraints on Tree-Level Higher Order Gravitational Couplings in Superstring Theory, Phys. Rev. Lett. 106 (2011) 111601 [arXiv:0910.0180] [InSPIRE].

[46] O. Schlotterer and S. Stieberger, Motivic Multiple Zeta Values and Superstring Amplitudes, J. Phys. A 46 (2013) 475401 [arXiv: 1205.1516] [InSPIRE].

[47] H. Kawai, D.C. Lewellen and S.H.H. Tye, A Relation Between Tree Amplitudes of Closed and Open Strings, Nucl. Phys. B 269 (1986) 1 [InSPIRE].

[48] D. Mazac and M.F. Paulos, The analytic functional bootstrap. Part I: $1 D$ CFTs and 2D S-matrices, JHEP 02 (2019) 162 [arXiv:1803.10233] [INSPIRE].

[49] B. Bellazzini, J. Elias Miró, R. Rattazzi, M. Riembau and F. Riva, Positive Moments for Scattering Amplitudes, arXiv:2011.00037 [INSPIRE].

[50] J.J.M. Carrasco, L. Rodina, Z. Yin and S. Zekioglu, Simple encoding of higher derivative gauge and gravity counterterms, Phys. Rev. Lett. 125 (2020) 251602 [arXiv:1910.12850] [INSPIRE]. 